\title{
Non-Higgsable clusters for 4D F-theory models
}

\author{
David R. Morrison ${ }^{a}$ and Washington Taylor ${ }^{b}$ \\ ${ }^{a}$ Departments of Mathematics and Physics, University of California, Santa Barbara, \\ Santa Barbara, CA 93106, U.S.A. \\ ${ }^{b}$ Center for Theoretical Physics, Department of Physics, Massachusetts Institute of Technology, \\ 77 Massachusetts Avenue, Cambridge, MA 02139, U.S.A. \\ E-mail: drm@math.ucsb.edu, wati@mit.edu
}

ABSTRACT: We analyze non-Higgsable clusters of gauge groups and matter that can arise at the level of geometry in 4D F-theory models. Non-Higgsable clusters seem to be generic features of F-theory compactifications, and give rise naturally to structures that include the nonabelian part of the standard model gauge group and certain specific types of potential dark matter candidates. In particular, there are nine distinct single nonabelian gauge group factors, and only five distinct products of two nonabelian gauge group factors with matter, including $\mathrm{SU}(3) \times \mathrm{SU}(2)$, that can be realized through $4 \mathrm{D}$ non-Higgsable clusters. There are also more complicated configurations involving more than two gauge factors; in particular, the collection of gauge group factors with jointly charged matter can exhibit branchings, loops, and long linear chains.

KEYwords: F-Theory, Gauge Symmetry, Supergravity Models, Superstring Vacua

ArXIV EPRINT: 1412.6112 


\section{Contents}

1 Introduction 1

2 Review of F-theory basics 4

3 Local conditions $\quad 6$

3.1 Derivation of local conditions 6

3.2 Summary of local conditions 8

3.3 Additional constraints 9

4 Warm-up: 6D non-Higgsable clusters $\quad 10$

$\begin{array}{lll}4.1 \text { Constraints on individual curves } & 10\end{array}$

4.2 Monodromy 11

$\begin{array}{lll}4.3 & \text { Matter } & 13\end{array}$

$\begin{array}{lll}4.4 & \text { Superconformal fixed points } & 13\end{array}$

$\begin{array}{ll}\text { 4.5 Clusters with multiple factors } & 13\end{array}$

5 4D non-Higgsable clusters with single gauge group factors $\quad 14$

$\begin{array}{lll}5.1 & \text { Possible single gauge factors } & 14\end{array}$

$\begin{array}{lll}5.2 \text { Matter } & 16\end{array}$

6 Products of two factors 18

7 More complicated "quiver diagrams" $\quad 19$

$\begin{array}{lll}7.1 & \text { Branchings } 20\end{array}$

7.2 Chains 20

$\begin{array}{lll}7.3 & \text { Loops } & 22\end{array}$

8 Conclusions $\quad 23$

8.1 Summary and open questions 23

8.2 Classifying Calabi-Yau fourfolds 25

$\begin{array}{lll}\text { 8.3 Physical consequences of non-Higgsable clusters } & 26\end{array}$

A The gauge algebra of maximally Higgsed models 29

\section{Introduction}

Many supersymmetric string theory compactifications contain "non-Higgsable" gauge groups that cannot be broken by charged matter in a way that preserves supersymmetry. In the simplest cases, the non-Higgsable gauge group is a single simple factor such as 
$\mathrm{SU}(3), \mathrm{SO}(8)$, or $E_{8}$ under which there are no charged matter fields. Such string vacua have long been known to arise in heterotic string compactifications, and in many cases have dual F-theory descriptions [1-3]; a simple set of examples are given by $6 \mathrm{D}$ supergravity theories arising from heterotic compactifications on K3 and dual F-theory compactifications on Hirzebruch surfaces $\mathbb{F}_{m}$. For example, a non-Higgsable $E_{8}$ arises in the $E_{8} \times E_{8}$ heterotic theory when all of the 24 instantons needed for tadpole cancellation in the 10D theory are placed in one of the two $E_{8}$ heterotic factors, corresponding on the F-theory side to compactification on $\mathbb{F}_{12}$. While in the simplest cases there is no charged matter, there are also cases where a gauge group is non-Higgsable even in the presence of charged matter. For example, in a $6 \mathrm{D}$ heterotic compactification where the numbers of instantons in the two $E_{8}$ factors are 5 and 19, corresponding on the F-theory side to a compactification on $\mathbb{F}_{7}$, there is a non-Higgsable gauge group $E_{7}$ carrying a half hypermultiplet in the $\mathbf{5 6}$ representation. This matter cannot be Higgsed in the low-energy theory since the D-term constraints cannot be satisfied by matter in a single real representation.

In heterotic constructions that use smooth bundles over smooth Calabi-Yau manifolds the non-Higgsable gauge groups contain only a single simple factor. In F-theory, however, many geometries give rise to non-Higgsable gauge groups with multiple factors and jointly charged matter. In [4], we performed a systematic analysis of the possible non-Higgsable structures that can arise in 6D F-theory compactifications, and identified all possible "nonHiggsable clusters" of gauge group factors connected by jointly charged matter that cannot be broken by Higgsing. The analysis was carried out by looking at configurations of intersecting curves on the (two complex dimensional) base surface, with each curve having a negative self-intersection. The complete list of non-Higgsable gauge groups in 6D F-theory models contains, in addition to the single group factors $\mathrm{SU}(3), \mathrm{SO}(8), F_{4}, E_{6}, E_{7}$, and $E_{8}$, the two product groups $G_{2} \times \mathrm{SU}(2)$ and $\mathrm{SU}(2) \times \mathrm{SO}(7) \times \mathrm{SU}(2)$, with non-Higgsable matter jointly charged under the adjacent factors in each gauge group. ${ }^{1}$

In this paper we initiate a systematic analysis of non-Higgsable clusters for 4D F-theory models. Non-Higgsable clusters give rise to gauge groups and matter at generic points in the moduli spaces of Calabi-Yau fourfolds over many bases that can be used for F-theory compactification. From our current understanding of the space of elliptically fibered CalabiYau manifolds, it seems that in fact the vast majority of F-theory compactifications will have such structure. Non-Higgsable clusters can give rise to the nonabelian part of the standard model [5], as well as decoupled or weakly interacting sectors that have a natural possible interpretation as dark matter. In section 8.3, we comment on some aspects of these constructions that may be relevant to phenomenology.

There are several issues that make the analysis of non-Higgsable clusters, and F-theory vacua in general, more complex for four-dimensional models than for six-dimensional models. In six dimensions the geometric complex structure moduli space of an elliptically fibered Calabi-Yau threefold matches with a continuous moduli space of flat directions in the corresponding $6 \mathrm{D}$ supergravity theory, so that there is a close correspondence between

\footnotetext{
${ }^{1}$ The non-Higgsable structure imposed from geometry determines only the gauge algebra, so that these groups may in principle be reduced through a quotient by a finite subgroup in some cases.
} 
the structure of the physical theory and the geometric data of F-theory (see for example [6]). In four dimensions this connection is obscured by the presence of a superpotential that lifts some of the flat directions. Viewing F-theory as dual to a limit of M-theory, the superpotential is produced by G-flux on a Calabi-Yau fourfold (see [7] for an introductory review). There are also additional degrees of freedom on the world-volume of IIB seven-brane configurations that are as yet not well understood or incorporated into the F-theory context. Even for perturbative (e.g. SU $(N)$ ) seven-brane stacks, off-diagonal excitations of the world-volume adjoint scalar fields encode expansion of $\mathrm{D} p$-branes into higher-dimensional $\mathrm{D}(p+2 k)$-branes [8-11]; these degrees of freedom are not encompassed in the complex structure moduli of the elliptically fibered Calabi-Yau used for F-theory and have been studied in that context as "T-branes" [12-15]. While such excitations can be described locally, unlike for the complex structure degrees of freedom in F-theory which have a global characterization in terms of Weierstrass models there is no analogous general global formulation of the full set of open string degrees of freedom associated with perturbative brane configurations on a general compact space (see e.g. [16, 17] for some initial efforts in this direction, and [18-20] for more recent developments and further references). For nonperturbative seven-brane configurations associated with exceptional groups, the open string dynamics is even less transparent from the F-theory complex structure point of view.

The effects of G-flux and additional degrees of freedom can not only lift flat directions in the moduli space, but can also modify the spectrum of the theory. On the one hand, the potential produced by G-flux can drive the theory to a point of enhanced symmetry, while on the other hand flux in the world-volume fields on a set of seven-branes can also break the apparent geometric symmetry to a smaller group. G-flux also affects the matter spectrum of the theory, and can give rise to chiral matter although the underlying CalabiYau geometry in the F-theory picture describes only non-chiral $(4 \mathrm{D} \mathcal{N}=2)$ matter. Some of these issues are discussed in more detail in [5]. Though there has been substantial work on various aspects of G-flux in 4D F-theory compactifications (see for example [21$27]$ ), there is still no completely general way of analyzing these effects in an arbitrary 4D compactification. In this paper, we focus only on the underlying geometry of the F-theory compactification, in particular on the continuous moduli space of complex structures for a given elliptically fibered Calabi-Yau fourfold parameterized by a Weierstrass model. When we refer to the geometric gauge group and geometric matter, we refer only to the gauge group and non-chiral matter associated with the singularities of the Weierstrass model. This analysis thus gives only a first-order picture of the space of possibilities that can exist in complete F-theory models. To determine the actual physical gauge group and matter the further incorporation of G-flux effects is necessary, and we leave this further analysis to future work. Another complication in the analysis of $4 \mathrm{D}$ F-theory models is the presence of codimension three loci where the Weierstrass coefficients $f, g$ vanish to degrees $(4,6)$. As we discuss in the next section, it is not yet understood whether such singularities pose a problem for consistency of $4 \mathrm{D}$ F-theory models, and we include vacua with such loci in the analysis here.

After a brief review of some basic aspects of F-theory in section 2, we begin in section 3 with a general set of formulae that can be used to give a lower bound for the orders 
of vanishing of the Weierstrass coefficients $f$ and $g$ over any given divisor in a complex threefold base. These formulae control the local singularity structure of the Weierstrass model and determine the factors that can appear in a non-Higgsable cluster. In section 4, as a warm-up exercise we use a simplified version of the local divisor formulae to describe non-Higgsable clusters in 6D theories, and reproduce the results of [4] in a simple and direct way. We then proceed in section 5 and the following sections to analyze the local structure of $4 \mathrm{D}$ clusters using the general formulae. We find a rich range of behavior, including branchings, loops, and long linear chains of connected gauge group factors. We conclude in section 8 with a discussion of some of the possible applications of $4 \mathrm{D}$ non-Higgsable clusters.

\section{Review of F-theory basics}

Here we summarize a few of the basic features of F-theory that are central to the analysis of this paper. More comprehensive reviews can be found in [7, 28, 29].

We consider F-theory as a nonperturbative formulation of type IIB string theory. A supersymmetric F-theory compactification to $10-2 n$ dimensions is defined by a complex $n$-fold base $B_{n}$ that supports an elliptic fibration with section $\pi: X \rightarrow B_{n}$ where the total space $X$ is a Calabi-Yau $(n+1)$-fold. The data of such an elliptic fibration can be described by a Weierstrass model [30]

$$
y^{2}=x^{3}+f x+g,
$$

where $f, g$ are sections of line bundles $\mathcal{O}(-4 K), \mathcal{O}(-6 K)$ over the base $B_{n}$, with $-K$ the anti-canonical class on $B_{n}$. The Weierstrass parameters $f$ and $g$ can be described in terms of polynomials of fixed degrees in a local coordinate system on $B_{n}$. The (geometric) gauge group of the corresponding supergravity theory is determined by the codimension one singularity structure of the Weierstrass model, where the discriminant $\Delta=4 f^{3}+27 g^{2}$ vanishes. When $f, g$, and $\Delta$ vanish to certain orders on a divisor (i.e., a codimension one algebraic subspace) then the total space of the elliptic fibration is singular, and can be viewed as a degenerate limit of a smooth Calabi-Yau manifold; in IIB language the singularities can be interpreted in terms of coincident seven-branes (but of more general types than occur in the perturbative IIB string). In either picture, the physical result is the appearance of a nonabelian gauge symmetry in the supergravity theory. The classification of codimension one singularities, following Kodaira [31, 32], is listed in table 1, along with the resulting gauge algebra factors (which are inferred from gauge symmetry enhancement in M-theory [33, 34]). In some cases, for compactifications to six dimensions or fewer the gauge group depends not only on the orders of vanishing of $f, g, \Delta$, but also on the more detailed monodromy structure of the singularity locus $[3,31,32,35-37]$. The (geometric) matter content of the theory is determined by the codimension two singularity locus on the base. In simple cases, the representation content of the matter is determined in a simple fashion from the enhancement of the Kodaira singularity type on the singular codimension two locus [35, 38], but more complicated matter representations can also arise. A complete dictionary between codimension two singularities and matter representations has not yet been developed, though a number of recent works have made progress in this 


\begin{tabular}{|c|c|c|c|c|c|}
\hline Type & $\operatorname{ord}(f)$ & $\operatorname{ord}(g)$ & $\operatorname{ord}(\Delta)$ & singularity & nonabelian symmetry algebra \\
\hline $\mathrm{I}_{0}$ & $\geq 0$ & $\geq 0$ & 0 & none & none \\
$\mathrm{I}_{n}$ & 0 & 0 & $n \geq 2$ & $A_{n-1}$ & $\mathfrak{s u}(n)$ or $\mathfrak{s p}(\lfloor n / 2\rfloor)$ \\
$\mathrm{II}$ & $\geq 1$ & 1 & 2 & none & none \\
$\mathrm{III}$ & 1 & $\geq 2$ & 3 & $A_{1}$ & $\mathfrak{s u}(2)$ \\
$\mathrm{IV}$ & $\geq 2$ & 2 & 4 & $A_{2}$ & $\mathfrak{s u}(3)$ or $\mathfrak{s u}(2)$ \\
$\mathrm{I}_{0}^{*}$ & $\geq 2$ & $\geq 3$ & 6 & $D_{4}$ & $\mathfrak{s o}(8)$ or $\mathfrak{s o}(7)$ or $\mathfrak{g}_{2}$ \\
$\mathrm{I}_{n}^{*}$ & 2 & 3 & $n \geq 7$ & $D_{n-2}$ & $\mathfrak{s o}(2 n-4)$ or $\mathfrak{s o}(2 n-5)$ \\
$\mathrm{IV}^{*}$ & $\geq 3$ & 4 & 8 & $\mathfrak{e}_{6}$ & $\mathfrak{e}_{6}$ or $\mathfrak{f}_{4}$ \\
$\mathrm{III}^{*}$ & 3 & $\geq 5$ & 9 & $\mathfrak{e}_{7}$ & $\mathfrak{e}_{7}$ \\
$\mathrm{II}^{*}$ & $\geq 4$ & 5 & 10 & $\mathfrak{e}_{8}$ & $\mathfrak{e}_{8}$ \\
\hline non-min & $\geq 4$ & $\geq 6$ & $\geq 12$ & \multicolumn{2}{|c|}{ does not occur for susy vacua } \\
\hline
\end{tabular}

Table 1. Table of codimension one singularity types for elliptic fibrations and associated nonabelian symmetry algebras. In cases where the algebra is not determined uniquely by the degrees of vanishing of $f, g$, the precise gauge algebra is fixed by monodromy conditions that can be identified from the form of the Weierstrass model.

direction [37, 39-47]. For the purposes of this paper, the most relevant fact is that, in general, matter arises at codimension two loci within codimension one divisors carrying gauge group factors, where the degrees of vanishing of $f, g$, and/or $\Delta$ are enhanced. In particular, when two divisors each carry a gauge group factor, and they intersect along a codimension two locus (a set of points in the case of $6 \mathrm{D}$ compactifications, or a complex curve in the case of $4 \mathrm{D}$ compactifications), then there is generally (geometric) matter that carries a charge under both of the gauge group factors.

A non-Higgsable gauge group factor arises on a given divisor $D$ when all sections $f$ of $\mathcal{O}(-4 K)$ and all sections $g$ of $\mathcal{O}(-6 K)$ vanish to orders $\phi \geq 1, \gamma \geq 2$ respectively on $D$. In such a situation, the orders of vanishing $\phi, \gamma$ force a gauge group factor according to the Kodaira conditions in table 1. Note that only certain gauge groups can be forced to appear in this way. In particular, type $\mathrm{I}_{n}$ and type $\mathrm{I}_{n}^{*}$ singularities with $n>0$ cannot be forced to arise in a generic Weierstrass model over any base.

If $f, g$ vanish to orders $(4,6)$ on a divisor, then there is a "non-minimal singularity" that cannot be resolved to give a total space that is Calabi-Yau (and hence the data does not describe a supersymmetric vacuum). If $f, g$ vanish to orders $(4,6)$ on a codimension two locus in the base, then there is again a non-minimal singularity. By blowing up the codimension two locus in the base a new base arises with a reduced degree of singularity, so that the total space of the fibration may either be resolvable into a Calabi-Yau directly, or after further blowups. It is also possible to describe the structure associated with a $(4,6)$ vanishing on a codimension two locus in terms of a superconformal field theory [48]; while such field theories have been the subject of some recent work [49-53], we do not investigate such structure here. In $4 \mathrm{D}$ models, the situation is less clear when $f, g$ vanish to degrees $(4,6)$ at a codimension three locus (point). At such points, like at $(4,6)$ codimension two loci, it seems that extra massless states appear in the theory [54]; the degree of vanishing is not sufficient at such points, however, to lead directly to a blowup of the point — for this 
we would need additional vanishing to order $(8,12)$. Thus, while it is possible that there is some problem or inconsistency in models with such codimension three singularities, it is also plausible that such models represent perfectly acceptable F-theory vacua. ${ }^{2}$ We do not try to resolve this question in this paper, but we do note some circumstances when this issue may affect some of the structures we describe for 4D non-Higgsable clusters.

\section{Local conditions}

Classifying the possible non-Higgsable clusters that can arise in F-theory compactifications to four dimensions is more difficult than for compactifications to six dimensions, even at the level of pure geometry. The approach used in [4] to classify non-Higgsable clusters on base surfaces incorporated a method known as the Zariski decomposition, whose generalization to three-dimensional bases has many complications [55]. Thus, we develop here some general local methods for placing constraints on the possible structure of non-Higgsable clusters for 4D F-theory models.

For a local or global base geometry with a toric description, it is straightforward to use the lattice of monomials dual to the lattice containing the toric fan [56] to compute the orders of vanishing of $f, g$ on any given divisor in a generic Weierstrass model. This method is described explicitly in [57] for base surfaces (where it was used to analyze the set of all toric bases that support elliptically fibered Calabi-Yau threefolds), and in [58] for threefold bases. We use this approach for explicit calculations in some specific examples in this paper, complementing the general methods developed in this section.

In section 3.1, we derive local conditions that can be used to show that $f, g$ have certain minimal orders of vanishing on divisors on a completely general base $B$. The results of this analysis are summarized in section 3.2 in a succinct fashion useful for explicit computations.

\subsection{Derivation of local conditions}

In [58], a general class of $4 \mathrm{D}$ F-theory models were considered where the base $B_{3}$ had the structure of a $\mathbb{P}^{1}$ bundle over a complex surface $B_{2}$, constructed from the projectivization of a line bundle $\mathcal{L}$ over $B_{2}$. As described in that paper, when there is a good coordinate $z$ in an open region containing a section $\Sigma$ of the $\mathbb{P}^{1}$ bundle (as is true, for example, when $B_{2}$ is toric), there is a series expansion $f=\hat{f}_{0}+\hat{f}_{1} z+\hat{f}_{2} z^{2}+\cdots$ whose coefficients $\hat{f}_{k}$ restrict to sections of $\mathcal{O}_{\Sigma}\left(-4 K_{\Sigma}-(4-k) T\right)$ on $\Sigma$, and similarly $\left.\hat{g}_{k}\right|_{\Sigma} \in \Gamma\left(\mathcal{O}_{\Sigma}\left(-6 K_{\Sigma}-(6-k) T\right)\right)$, where $T=c_{1}(\mathcal{L})$ characterizes the "twist" of the line bundle $\mathcal{L}$, and $K_{\Sigma}$ is the canonical class of the complex surface $\Sigma \cong B_{2} \subset B_{3}$. The key way that this expansion is used is in demonstrating that certain of these coefficients must vanish upon restriction to $\Sigma$ (showing that $f$ or $g$ must vanish to certain orders) by checking that the corresponding line bundles on $\Sigma$ have no non-vanishing sections at all.

This characterization of vanishing conditions for $f$ and $g$ can be made more precise and generalized to an arbitrary divisor in a general base $B$ (of any dimension). In the above description, the local geometry around the divisor $\Sigma$ is characterized by the normal

\footnotetext{
${ }^{2}$ We thank Antonella Grassi for discussions on this point.
} 
line bundle, which is $N_{\Sigma}=N_{\Sigma / B_{3}}=-T$, so the conditions on $f$ and $g$ depend only on the local geometry and not on the global structure as a $\mathbb{P}^{1}$ bundle. In general, therefore, if we have a base $B$ containing an effective divisor $D$, we initially have

$$
\begin{aligned}
& \left.f\right|_{D} \in \Gamma\left(\mathcal{O}_{D}\left(-4 K_{D}+4 N_{D}\right)\right) \\
& \left.g\right|_{D} \in \Gamma\left(\mathcal{O}_{D}\left(-6 K_{D}+6 N_{D}\right)\right),
\end{aligned}
$$

where $K_{D}$ and $N_{D}=N_{D / B}$ are the canonical and normal line bundles for $D \subset B$, and we have used the adjunction formula, which tells us that $-\left.K_{B}\right|_{D}=-K_{D}+N_{D}$.

There are exact sequences that help to measure the vanishing of $f$ and $g$ along $D$ :

$$
\begin{aligned}
& 0 \rightarrow \mathcal{O}\left(-4 K_{B}-D\right) \rightarrow \mathcal{O}\left(-4 K_{B}\right) \rightarrow \mathcal{O}_{D}\left(-4 K_{D}+4 N_{D}\right) \rightarrow 0 \\
& 0 \rightarrow \mathcal{O}\left(-6 K_{B}-D\right) \rightarrow \mathcal{O}\left(-6 K_{B}\right) \rightarrow \mathcal{O}_{D}\left(-6 K_{D}+6 N_{D}\right) \rightarrow 0
\end{aligned}
$$

Thanks to these sequences, if $\left.f\right|_{D}$ vanishes then we can write $f=\hat{f}_{1} z$ with $\hat{f}_{1}$ a section of $\mathcal{O}\left(-4 K_{B}-D\right)$. Similarly, if $\left.g\right|_{D}$ vanishes then we can write $g=\hat{g}_{1} z$ with $\hat{g}_{1}$ a section of $\mathcal{O}\left(-6 K_{B}-D\right)$.

We can continue, and try to detect if $f$ or $g$ vanishes to order 2. For this purpose, we use the pair of exact sequences

$$
\begin{aligned}
& 0 \rightarrow \mathcal{O}\left(-4 K_{B}-2 D\right) \rightarrow \mathcal{O}\left(-4 K_{B}-D\right) \rightarrow \mathcal{O}_{D}\left(-4 K_{D}+3 N_{D}\right) \rightarrow 0 \\
& 0 \rightarrow \mathcal{O}\left(-6 K_{B}-2 D\right) \rightarrow \mathcal{O}\left(-6 K_{B}-D\right) \rightarrow \mathcal{O}_{D}\left(-6 K_{D}+5 N_{D}\right) \rightarrow 0
\end{aligned}
$$

To understand these sequences it is helpful to recall that $\mathcal{O}_{D}(-D)$ is an alternate way of writing the line bundle $\mathcal{O}_{D}\left(-N_{D}\right)$, and we have used this equivalence in the exact sequence. Note that $\hat{f}_{1}$ or $\hat{g}_{1}$, when they exist, are sections of the middle term in the exact sequence; we restrict them to $D$, and if one of them is zero, then we will be able to write $\hat{f}_{1}=\hat{f}_{2} z$ (or $\hat{g}_{1}=\hat{g}_{2} z$ ), i.e., $f=\hat{f}_{2} z^{2}$ (or $g=\hat{g}_{2} z^{2}$ ). This happens if and only if $f$ (respectively $g$ ) vanishes to order at least 2 along $D$.

We now see the general pattern: if $f$ vanishes to order at least $k$ then we can write $f=\hat{f}_{k} z^{k}$ with $\hat{f}_{k}$ a section of $\mathcal{O}\left(-4 K_{B}-k D\right)$ and restrict $\hat{f}_{k}$ to $D$. The corresponding exact sequence is

$$
0 \rightarrow \mathcal{O}\left(-4 K_{B}-(k+1) D\right) \rightarrow \mathcal{O}\left(-4 K_{B}-k D\right) \rightarrow \mathcal{O}\left(-4 K_{D}+(4-k) N_{D}\right) \rightarrow 0 .
$$

The restriction vanishes if and only if $f$ vanishes to order at least $k+1$ along $D$, and in that case we can write $\hat{f}_{k}=\hat{f}_{k+1} z$ so that $f=\hat{f}_{k+1} z^{k+1}$.

Similarly, if $g$ vanishes to order at least $k$ then we can write $g=\hat{g}_{k} z^{k}$ with $\hat{g}_{k}$ a section of $\mathcal{O}\left(-6 K_{B}-k D\right)$ and restrict $\hat{g}_{k}$ to $D$. The corresponding exact sequence is

$$
0 \rightarrow \mathcal{O}\left(-6 K_{B}-(k+1) D\right) \rightarrow \mathcal{O}\left(-6 K_{B}-k D\right) \rightarrow \mathcal{O}\left(-6 K_{D}+(6-k) N_{D}\right) \rightarrow 0 .
$$

The restriction vanishes if and only if $g$ vanishes to order at least $k+1$ along $D$, and in that case we can write $\hat{g}_{k}=\hat{g}_{k+1} z$ so that $g=\hat{g}_{k+1} z^{k+1}$. 
More generally, given a set of effective divisors $D_{a}$ in $B$ together with the information that $f$ vanishes on $D_{a}$ to order at least $\phi_{a}$ and $g$ vanishes on $D_{a}$ to order at least $\gamma_{a}$, then we can write

$$
\begin{aligned}
& f=f^{[a]} \prod_{b \neq a} z_{b}^{\phi_{b}} \\
& g=g^{[a]} \prod_{b \neq a} z_{b}^{\gamma_{b}}
\end{aligned}
$$

where $z_{b}$ is a local coordinate vanishing on $D_{b}$.

We now go through the same reasoning with analogous exact sequences, starting from $-4 K_{B}-\sum_{b \neq a} \phi_{b} D_{b}$ instead of $-4 K_{B}$, and $-6 K_{B}-\sum_{b \neq a} \gamma_{b} D_{b}$ instead of $-6 K_{B}$, to determine the orders of vanishing of $f^{[a]}$ and $g^{[a]}$. If the order of vanishing is at least $k$, then the restricted leading coeffient lies in

$$
\left.f_{k}^{[a]}\right|_{D_{a}} \in \Gamma\left(\mathcal{O}_{D_{a}}\left(-4 K^{(a)}+(4-k) N^{(a)}-\sum_{b \neq a} \phi_{b} C_{a b}\right)\right)
$$

or

$$
\left.g_{k}^{[a]}\right|_{D_{a}} \in \Gamma\left(\mathcal{O}_{D_{a}}\left(-6 K^{(a)}+(6-k) N^{(a)}-\sum_{b \neq a} \gamma_{b} C_{a b}\right)\right),
$$

where $C_{a b}=D_{a} \cap D_{b}$, considered as a curve in $D_{a}$. The terms proportional to $C_{a b}$ arise because the vanishing of $f, g$ around $D_{b}$ appear on $D_{a}$ as additional vanishings on the curves $C_{a b}$.

The properties of the bundles in (3.11) and (3.12) can be used to determine the minimal possible orders of vanishing $\phi_{a}, \gamma_{a}$ of $f, g$ on each divisor $D_{a}$ in a self-consistent fashion. By using the fact that e.g. $\left.f_{k}^{[a]}\right|_{D_{a}}$ must vanish if the line bundle of which it is a section corresponds to a non-effective divisor on $D_{a},(3.11)$ and (3.12) specify a collection of bundles which can be checked for the existence of non-zero sections, and if those sections are absent, the corresponding leading coeffients must vanish (i.e., the order of vanishing will be greater than might have been expected).

For the monodromy conditions associated with each divisor, and to identify the complete (geometric) matter content, we need to consider these coefficients more generally as sections of (3.1), (3.2) that vanish to orders $\phi_{b}, \gamma_{b}$ on $C_{a b}$, and we use $\hat{f}_{k}^{[a]}\left|{ }_{D_{a}}, \hat{g}_{k}^{[a]}\right|_{D_{a}}$ in such situations.

\subsection{Summary of local conditions}

We summarize here the constraints derived in the previous section and define some notation that will be useful for explicit calculations. For a compactification of F-theory on a base $B$, for each effective divisor $D_{a}$ in $B$ we define corresponding families of divisors

$$
\begin{aligned}
& F_{k}^{(a)}=-4 K^{(a)}+(4-k) N^{(a)}-\sum_{b \neq a} \phi_{b} C_{a b} \\
& G_{k}^{(a)}=-6 K^{(a)}+(6-k) N^{(a)}-\sum_{b \neq a} \gamma_{b} C_{a b} .
\end{aligned}
$$


Here, as above, $-K^{(a)}, N^{(a)}$ are the divisors associated with the anti-canonical and normal line bundles to $D_{a}, \phi_{a}, \gamma_{a}$ are the orders of vanishing of $f, g$ on $D_{a}$, and $C_{a b}$ is the curve $D_{a} \cap D_{b}$ considered as a divisor class on $D_{a}$.

When there is no effective divisor in any of the divisor classes $F_{j}^{(a)}$ for $j=0,1, \ldots, k-1$, then $f$ must vanish to at least order $k$ on $D_{a}$. Similarly, when there is no effective divisor in any of the divisor classes $G_{j}^{(a)}$ for $j=0,1, \ldots, k-1$, then $g$ must vanish to at least order $k$ on $D_{a}$. This determines a set of conditions on the vanishing orders of $f, g$ on different divisors in the base that must be satisfied in a self-consistent fashion. Note that these conditions determine a minimum order of vanishing of $f, g$ on each divisor through the structure of the local geometry. We have not ruled out the possibility that further nonlocal structure may force $f, g$ to vanish to higher orders in some circumstances.

In the following sections we use the divisors specified in (3.13) and (3.14) to analyze various situations in which the vanishing of $f, g$ to particular orders guarantees the existence of non-Higgsable clusters of different kinds in Calabi-Yau threefolds and fourfolds corresponding to F-theory compactifications to $6 \mathrm{D}$ and $4 \mathrm{D}$ respectively.

In general the restriction of the leading non-vanishing term in an expansion of $f$ around the divisor $D_{a}$ can be described as a section of the line bundle over $D_{a}$ associated with $F_{k}$

$$
f_{k}^{(a)}=\left.f_{k}^{[a]}\right|_{D_{a}} \in \Gamma\left(\mathcal{O}_{D_{a}}\left(F_{k}\right)\right), \quad k=\phi_{a}
$$

and the restricted leading term in $g$ can similarly be described as a section of

$$
g_{k}^{(a)}=\left.g_{k}^{[a]}\right|_{D_{a}} \in \Gamma\left(\mathcal{O}_{D_{a}}\left(G_{k}\right)\right), \quad k=\gamma_{a} .
$$

Note that for a general base these equations are only meaningful for the first non-vanishing term in each of $f$ and $g$, though in special cases such as toric bases where there are good global coordinates, these expressions are valid for all $k$.

As discussed above, when determining monodromy conditions and matter content, it is useful to consider the leading terms $\hat{f}_{k}^{(a)}=\left.\hat{f}_{k}^{[a]}\right|_{D_{a}}, \hat{g}^{(g)}=\left.\hat{g}_{k}^{[a]}\right|_{D_{a}}$ as sections of the line bundles $\Gamma\left(\mathcal{O}_{D_{a}}\left(\hat{F}_{k}\right)\right), \Gamma\left(\mathcal{O}_{D_{a}}\left(\hat{G}_{k}\right)\right)$, with $\hat{F}_{k}=-4 K^{(a)}+(4-k) N^{(a)}, \hat{G}_{k}=-6 K^{(a)}+$ $(6-k) N^{(a)}$.

\subsection{Additional constraints}

We conclude this section by briefly mentioning a further local constraint that is not used directly in the analysis of this paper, but which may be useful in further analyzing the set of possible local divisor configurations and associated non-Higgsable clusters in general F-theory models.

In addition to the constraints described in the preceding sections, we have the geometric constraint

$$
N^{(b)} \cdot C_{b a}=C_{a b} \cdot C_{a b} .
$$

As can be inferred from the notation, the intersection on the left is carried out within $D_{b}$, while that on the right is in $D_{a}$.

This constraint follows from a general fact about intersection theory: if $D_{1}, D_{2}$, and $D_{3}$ are three divisors, then $D_{1} \cdot D_{2} \cdot D_{3}$ can be computed as an intersection of two divisors 
on $D_{3}$, namely, the intersection of the divisors $\left.D_{1}\right|_{D_{3}}$ and $\left.D_{2}\right|_{D_{3}}$. Permuting the $D_{i}$ 's gives multiple ways to compute the same intersection property. To apply this to derive (3.17), we consider the intersection product $D_{b} \cdot D_{b} \cdot D_{a}$. On the one hand, this can be evaluated on $D_{b}$ as the intersection of $\left.D_{b}\right|_{D_{b}}=N_{D}$ with $\left.D_{a}\right|_{D_{b}}=C_{b a}$. On the other hand, the same triple intersection can be evaluated on $D_{a}$ as the intersection of $\left.D_{b}\right|_{D_{a}}$ with $D_{b} \mid D_{a}$, i.e., as $C_{a b} \cdot C_{a b}$.

In the toric situation, the relation (3.17) follows directly from the structure of the fan for a toric threefold. Assuming that the geometry is smooth, and taking a choice of coordinates where $D_{a}, D_{b}$ are associated with rays $v_{a}=(0,0,1), v_{b}=(0,1,0)$, and there are $3 \mathrm{D}$ cones connecting these two rays to the rays $v_{c}=(1,0,0)$ and $v_{d}=(-1, y, z)$, we see that both sides of (3.17) are identified with the value $-y$. In the left-hand side, we have $N^{(b)}=-y C_{b d}$ and $C_{b d} \cdot C_{b a}=1$, so $N^{(b)} \cdot C_{b a}=-y$, and on the right-hand side we have the same result since $C_{a b}$ is a curve of self-intersection $-y$ from the fact that on projection to the plane $z=0, y v_{b}=v_{c}+v_{d}$.

\section{Warm-up: 6D non-Higgsable clusters}

As an illustration of how the constraints derived in the previous section can be used to characterize non-Higgsable clusters, we begin as a warm-up exercise with the $6 \mathrm{D}$ case. A complete classification of non-Higgsable clusters for 6D F-theory compactifications was given in [4]. Here we show how these results can be reproduced easily using the constraint equations derived in the previous section.

\subsection{Constraints on individual curves}

In six dimensions, we are concerned with Calabi-Yau threefolds that are elliptically fibered over a complex base surface $B_{2}$. In this situation, the divisors that support codimension one singularities of the elliptic fibration associated with gauge group factors are curves, and codimension two singularities are associated with points. This simplifies the analysis significantly, since all points on a curve represent the same homology class, so we can represent all divisors on a curve simply as an integer in $\mathbb{Z}$. Specializing the discussion of the previous section to the case of a base of dimension 2 , and denoting the divisors by $C_{a}$ since they are now curves on the base, we find

$$
\begin{aligned}
& F_{k}^{(a)}=-4 K^{(a)}+(4-k) N^{(a)}-\sum_{b} \phi_{b} Z_{a b}, \\
& G_{k}^{(a)}=-6 K^{(a)}+(6-k) N^{(a)}-\sum_{b} \gamma_{b} Z_{a b} .
\end{aligned}
$$

Here, as before, $K^{(a)}$ is the canonical class of $C_{a}, N^{(a)}$ is the class of the normal bundle, while $Z_{a b}$ is the intersection of $C_{a}$ with $C_{b}$, considered as a zero-cycle on $C_{a}$. (When $C_{a}$ is a rational curve, the only thing that matters about this zero-cycle is its degree $p_{a b}=\operatorname{deg} Z_{a b}$, which is the intersection number $C_{a} \cdot C_{b}$ ).

We begin by noting that if the anti-canonical class $-K_{C}=-K^{(a)}$ is not effective (i.e. a nonnegative integer class) for a given curve $C=C_{a}$, then (dropping the superscript $(a)$ 
henceforth on $f_{k}, g_{k}$, which we take to be assumed for any given curve $C$ ) $F_{k}, G_{k}$ could not be effective and $f_{k}, g_{k}$ would vanish for all $k$ unless $N_{C}$ were effective, in which case no $f_{k}$, $g_{k}$ could be non-vanishing unless the same were true of $f_{0}, g_{0}$. Similarly, if $-K_{C}=0$ then either all $f_{k}, g_{k}$ can be nonvanishing or none can. Since $-K_{C}=2-2 g$ on an irreducible curve of genus $g$, this leads us to the conclusion that there cannot be a non-Higgsable cluster on any curve of higher genus; this was shown from a different point of view in [4].

We assume then that all irreducible curves $C_{a}$ supporting a non-Higgsable cluster are rational curves (i.e., equivalent to $\mathbb{P}^{1}$ ). We have then $-K^{(a)}=2$, and $N^{(a)}=C_{a} \cdot C_{a}$ is the self-intersection of $C_{a}$.

Consider for example the case where $C_{a}$ is a curve of self-intersection -2 . In this case, $\operatorname{deg} F_{k}=2 k-\phi, \operatorname{deg} G_{k}=2 k-\gamma$, where $\phi=\sum_{b \neq a} \phi_{b} p_{a b}$. If $\phi=0$, so there is no forced vanishing of $f$ on any curves that intersect $C_{a}$, then $\operatorname{deg} F_{0}=\operatorname{deg} G_{0}=0$. We then have $f_{0} \in \mathcal{O}(0)=\mathbb{C}$, and similarly for $g_{0}$, so there is no forced vanishing of $f, g$ on $C_{a}$.

Now consider the case of a curve of self-intersection $N^{(a)}=-3$. In this case,

$$
\begin{aligned}
\operatorname{deg} F_{k} & =-4+3 k-\phi \\
\operatorname{deg} G_{k} & =-6+3 k-\gamma .
\end{aligned}
$$

It follows that $f_{0}, f_{1}, g_{0}, g_{1}$ must all vanish, so the curve $C_{a}$ must support a Kodaira type IV $(2,2,4)$ singularity. Furthermore, $g_{2}=\hat{g}_{2} \in \mathcal{O}(0)=\mathbb{C}$ (assuming $\phi=0$ ), which satisfies the monodromy condition so that the associated gauge group is $\mathrm{SU}(3)$. This analysis is essentially equivalent to the Zariski decomposition method used in [4], in which $-K$ is decomposed over the rationals, so that for a self-intersection -3 curve $C$, with $-K \cdot C=-1$, $-K=C / 3+X$ with $X(\mathbb{Q}$-)effective (actually nef) from which it follows that $-4 K$ contains two factors of $C$ as irreducible components, as does $-6 K$. The method of analysis used here, however, generalizes more readily to four-dimensional F-theory compactifications than the Zariski approach.

Systematically applying these methods for any irreducible rational curve $C_{a}$ of given self-intersection, the divisors $F_{k}, G_{k}$ are easily computed and determine the orders of vanishing of $f, g$ over the curve $C_{a}$, as tabulated in table 2. From the data in this table, we can determine many features of the gauge groups and matter that arise at generic points in complex structure moduli space for bases that contain one or more intersecting curves of negative self-intersection. In particular, we can determine the precise minimal gauge group, including effects of monodromy; we can ascertain the generic matter content; we can identify cases where there is a $(4,6)$ singularity at a point; and we can classify nonHiggsable clusters containing multiple gauge group factors. We describe a few details of each of these aspects in the following subsections.

\subsection{Monodromy}

In the cases of Kodaira singularities of types IV, $\mathrm{I}_{0}^{*}$, and $\mathrm{IV}^{*}$, the gauge group of the low-energy theory depends upon an additional monodromy condition; the Dynkin diagram describing the set of cycles produced when a codimension one singularity is resolved can be mapped to itself non-trivially under a closed path in the relevant divisor that goes around 


\begin{tabular}{|r|c|c|l|}
\hline$C \cdot C$ & Divisors $F_{k}$ & Divisors $G_{k}$ & singularity type \\
\hline-1 & $\operatorname{deg} F_{k}=4+k-\phi$ & $\operatorname{deg} G_{k}=6+k-\gamma$ & $(0,0,0)$ \\
-2 & $\operatorname{deg} F_{k}=2 k-\phi$ & $\operatorname{deg} G_{k}=2 k-\gamma$ & $(0,0,0)$ \\
-3 & $\operatorname{deg} F_{2+n}=2+3 n-\phi$ & $\operatorname{deg} G_{2+n}=3 n-\gamma$ & $(2,2,4) \Rightarrow \operatorname{IV}\left(\mathfrak{s u}_{3}\right)$ \\
-4 & $\operatorname{deg} F_{2+n}=4 n-\phi$ & $\operatorname{deg} G_{3+n}=4 n-\gamma$ & $(2,3,6) \Rightarrow \mathrm{I}_{0}^{*}\left(\mathfrak{s o}_{8}\right)$ \\
-5 & $\operatorname{deg} F_{3+n}=3+5 n-\phi$ & $\operatorname{deg} G_{4+n}=2+5 n-\gamma$ & $(3,4,8) \Rightarrow \mathrm{IV}^{*}\left(\mathfrak{f}_{4}\right)$ \\
-6 & $\operatorname{deg} F_{4}=2+6 n-\phi$ & $\operatorname{deg} G_{4+n}=6 n-\gamma$ & $(3,4,8) \Rightarrow \operatorname{IV}^{*}\left(\mathfrak{e}_{6}\right)$ \\
-7 & $\operatorname{deg} F_{3+n}=1+7 n-\phi$ & $\operatorname{deg} G_{5+n}=5+7 n-\gamma$ & $(3,5,9) \Rightarrow \operatorname{III}^{*}\left(\mathfrak{e}_{7}\right)$ \\
-8 & $\operatorname{deg} F_{3+n}=8 n-\phi$ & $\operatorname{deg} G_{5+n}=4+8 n-\gamma$ & $(3,5,9) \Rightarrow \operatorname{III}^{*}\left(\mathfrak{e}_{7}\right)$ \\
$-9 / 10 / 11$ & $\operatorname{deg} F_{4}=8-\phi$ & $\operatorname{deg} G_{5}=3 / 2 / 1-\gamma$ & $(4,5,10) \Rightarrow \operatorname{II}^{*}\left(\mathfrak{e}_{8}\right)$ \\
-12 & $\operatorname{deg} F_{4}=8-\phi$ & $\operatorname{deg} G_{5}=-\gamma$ & $(4,5,10) \Rightarrow \operatorname{II}^{*}\left(\mathfrak{e}_{8}\right)$ \\
\hline
\end{tabular}

Table 2. Table of degrees of divisors $F_{k}, G_{k}$ and resulting non-Higgsable singularity types on single rational curves $C$ of self-intersection -1 through -12 .

a codimension two singularity. The details of how the gauge group is determined in this case are worked out in [35, 37]; when considering the generic structure in the moduli space as is relevant for non-Higgsable clusters, the monodromy condition can be read off directly from the form of the leading terms in $f, g$ in the expansion around the divisor. These monodromy conditions on monomials for non-Higgsable clusters are described briefly in section 9 of [58], and analyzed and explained further in appendix A. These monodromy conditions are valid for F-theory compactifications in any dimension below eight, and will also be used in analyzing compactifications to $4 \mathrm{D}$ in later sections.

For type IV and $\mathrm{IV}^{*}$, the monodromy is determined by the leading coefficient in $g$. For a type IV codimension one singularity, if $\hat{g}_{2}$ is a perfect square, then there is no nontrivial monodromy and the gauge algebra is $\mathfrak{s u}_{3}$; otherwise it is $\mathfrak{s u}_{2}$. For $\hat{g}_{2}$ to generically be a perfect square, every section of $\mathcal{O}_{D}\left(-6 K_{D}+4 N_{D / B}\right)$ that comes from the restriction of a section of $\mathcal{O}_{B}\left(-6 K_{B}-2 D\right)$ must be the square of a section of $\mathcal{O}_{D}\left(-3 K_{D}+2 N_{D / B}\right)$. (In particular, it's not hard to see that the space of these sections can only be one-dimensional.) This is clearly the case for a -3 curve that does not intersect any other curves where $g$ vanishes $(\gamma=0)$, where $\hat{g}_{2} \in \mathcal{O}(0)$ so the non-Higgsable gauge group there has an algebra $\mathfrak{s u}_{3}$. Similarly, for a type $\mathrm{IV}^{*}$ singularity the gauge algebra is $\mathfrak{e}_{6}$ (no monodromy) if $\hat{g}_{4}$ is a perfect square, and $\mathfrak{f}_{4}$ otherwise. This allows us to immediately read off the $\mathfrak{f}_{4}$ and $\mathfrak{e}_{6}$ gauge algebras of the non-Higgsable cluster over curves of self-intersection $-5,-6$ respectively. For a non-Higgsable type $\mathrm{I}_{0}^{*}$ singularity, the gauge algebra is $\mathfrak{s o}_{8}$ (no monodromy) only when $\hat{f}_{2}, \hat{g}_{3}$ are both in one-dimensional spaces of sections $\Gamma(\mathcal{O}(2 X)), \Gamma(\mathcal{O}(3 X))$, and are proportional to second and third powers of some section $u$ in the one-dimensional space of sections $\Gamma(\mathcal{O}(X))$, where $X=-K_{D}+N_{D / B}$. In this case, the cubic $x^{3}+\hat{f}_{2} x+\hat{g}_{3}$ can be algebraically factorized to a product $(x-A)(x-B)(x-C)$ for generic choices of $f, g$. This condition is clearly satisfied for the non-Higgsable cluster over a -4 curve, where $X=0$. The remaining monodromy condition is that for a non-Higgsable type $\mathrm{I}_{0}^{*}$ singularity, we have a gauge algebra $\mathfrak{s o}_{7}$ when $x^{3}+\hat{f}_{2} x+\hat{g}_{3}$ factorizes into the product of a quadratic times a linear term for generic $f, g$. When $f$ and $g$ are generic so that maximal Higgsing has been 
done, this occurs only when $\hat{g}_{3}$ vanishes identically and $\hat{f}_{2}$ is not a perfect square; in all other cases the gauge algebra (of generic models) is $\mathfrak{g}_{2}$. The $\mathfrak{s o}_{7}$ condition does not occur for any of the non-Higgsable clusters over a single curve, but does occur over a combination of curves $-2,-3,-2$ as discussed below.

\subsection{Matter}

Matter can arise in F-theory constructions either from nonlocal structure, associated in $6 \mathrm{D}$ compactifications with the genus of the divisor on which a gauge group is supported, or from local structure associated with codimension two singularities. For gauge groups without monodromy, the presence of matter can be identified when there are codimension two loci on the gauge group divisor where the Kodaira singularity type is enhanced. A specific example of this can be seen for a -7 curve, where $F_{3}=1-\phi$ and $\hat{f}_{3} \in \mathcal{O}(1)$, so generically there is a point where $\hat{f}_{3}$ vanishes and the singularity type becomes $\mathrm{II}^{*}(4,5,10)$. This corresponds to the appearance of a half-hypermultiplet in the $\mathbf{5 6}$ representation. For the other single non-Higgsable gauge group factors without monodromy, such as $\mathfrak{s u}_{3}$ on a -3 curve, there are no points where the Kodaira singularity type is enhanced, since e.g. $g_{2} \in \Gamma(\mathcal{O}(-\gamma))=\mathbb{C}$ when $\gamma=0$. For a -5 curve, there are generically two points where $g_{4}$ vanishes. These, however, are the points around which there is monodromy; a double cover of $\mathbb{P}^{1}$ with two branch points is again a $\mathbb{P}^{1}$, and there is again no matter in this case (see e.g. [39, 59]).

\subsection{Superconformal fixed points}

Analogous to the appearance of matter, at loci on a II* curve where $g_{5}$ vanishes, there is a $(4,6,12)$ vanishing of $(f, g, \Delta)$. Such points correspond to theories where gravity is coupled to a superconformal field theory [48]; these are branch points in the moduli space that are associated with tensionless string transitions [60,61]. By blowing up the $(4,6)$ point in the base, one enters a different branch of the moduli space where the $6 \mathrm{D}$ theory has an extra tensor multiplet $[3,49]$. Such tensionless string transitions unify the space of all $6 \mathrm{D}$ F-theory compactifications into a single connected space $[6,62,63]$.

\subsection{Clusters with multiple factors}

From table 2 we can also determine the set of possible non-Higgsable clusters containing multiple intersecting curves of negative self-intersection, reproducing the results of [4]. Restricting attention to bases that do not include $(4,6)$ points, clearly there cannot be an intersection between two curves where the orders of vanishing of $f, g$ add to $(4,6)$ or more. This rules out any intersection between two curves each of self-intersection -4 or below. Even for two intersecting -3 curves, since $g$ vanishes on each to order at least two, we must have for each $\gamma \geq 2$, which implies $g_{2}=0$ on each, so there is a $(4,6)$ point at the intersection. Any intersection between a -3 curve and a curve of self-intersection -4 or below is even worse. So the only intersections that we need to consider for curves of self-intersection -2 or below are between $-2,-2$ or $-2,-3$ curves. Any combination of -2 curves alone cannot give rise to a non-Higgsable factor since we can have $f_{0}, g_{0} \in$ $\Gamma(\mathcal{O}(0))=\mathbb{C}$ on each -2 curve. Considering configurations with -3 and -2 curves, it is 
easy to check that the only nontrivial combinations giving non-Higgsable clusters are the $-2,-3$ and $-2,-2,-3$ combinations giving $\mathfrak{s u}_{2} \oplus \mathfrak{g}_{2}$ algebras and the $-2,-3,-2$ cluster that gives $\mathfrak{s u}_{2} \oplus \mathfrak{s o}_{7} \oplus \mathfrak{s u}_{2}$, as found in [4]. For example, on a -2 curve $C$ that intersects a -3 curve $D$, we must have $\phi_{C}, \gamma_{C} \geq 2$, which implies $\operatorname{ord}_{C} f, \operatorname{ord}_{C} g \geq 1$, which pushes $\gamma_{D} \geq 1$, so $g_{2}^{D}=0$, so $\gamma_{C} \geq 3$, and we have then type $\mathrm{I}_{0}^{*}$ and III singularities on the $-3,-2$ curves respectively, with generic monodromy on the $\mathrm{I}_{0}^{*}$ since $g_{3}^{D} \in \Gamma(\mathcal{O}(1))$ is nonzero and is not a constant. This reproduces the $\mathfrak{g}_{2} \oplus \mathfrak{s u}_{2}$ gauge algebra found in [4] for the $-3,-2$ non-Higgsable cluster. The story is similar the other cases. Note that for the $-2,-3,-2$ cluster, on the -3 curve we have $\gamma=4$, so $g_{3}=0$, while $\phi=2$, so $f_{2} \in \Gamma(\mathcal{O}(0))$ and $\hat{f}_{2} \in \Gamma\left(\mathcal{O}(2)\right.$ ), where $\hat{f}_{2}$ has two distinct roots (corresponding to the points of intersection with the two -2 curves) and is not a perfect square, so $x^{3}-\hat{f}_{2} x$ cannot factorize completely and the monodromy is $\mathfrak{s o}_{7}$. Further analysis of this type can be used to confirm the various combinations of non-Higgsable clusters that can be connected by -1 curves as enumerated in [4].

\section{$54 \mathrm{D}$ non-Higgsable clusters with single gauge group factors}

We now turn to F-theory compactifications to four dimensions, which involve compactification on Calabi-Yau fourfolds that are elliptically fibered over a threefold base $B_{3}$. While the story is in some ways parallel to that of six dimensions, there are a number of additional complications for four-dimensional theories, and the set of possible non-Higgsable clusters seems to be substantially richer than for $6 \mathrm{D}$ models. One issue that makes a general analysis of non-Higgsable clusters in threefold bases more complicated than in twofold bases is the wide range of possible surfaces that can arise as divisors in the threefold base. In the case of Calabi-Yau threefolds, as discussed in the previous section, curves in the twofold base are classified by genus, and the only curve topology that can support a non-Higgsable cluster is a $\mathbb{P}^{1}$. In base threefolds, on the other hand, a vast range of surfaces can be realized as divisors. Restricting to toric surfaces alone, each of the 61,539 toric surfaces enumerated in [57] can arise as a codimension one divisor in a threefold base that supports an elliptic fibration (in the simplest case by taking a product with $\mathbb{P}^{1}$ ). Hundreds of distinct choices of these divisor geometries can support non-Higgsable clusters [67]. A complete analysis of all algebraic surfaces that can act as divisors supporting non-Higgsable clusters represents a substantial project for future investigation.

The simplest non-Higgsable gauge groups are single nonabelian factors. We describe the possible single factor groups in section 5.1. In section 5.2 we describe the possible appearance (at the level of geometry) of matter localized on curves in the threefold base, which presents richer possibilities than in $6 \mathrm{D}$.

\subsection{Possible single gauge factors}

The set of possible isolated simple gauge algebras for $4 \mathrm{D}$ models is basically the same as for $6 \mathrm{D}$, with the additional possibilities of $\mathfrak{s u}_{2}$ and $\mathfrak{s o}_{7}$. The only possibilities from the Kodaira table that are ruled out, in fact, are those where the order of vanishing of $\Delta$ exceeds that 
determined by $f, g$ :

$$
\operatorname{ord}(\Delta)>\max (3 \operatorname{ord}(f), 2 \operatorname{ord}(g)) .
$$

For this to occur, we would need to have a cancellation between the leading terms in $\Delta=4 f^{3}+27 g^{2}$. But such a cancellation cannot occur between generic sections, since we can always multiply $f$ and $g$ by different constant complex factors and preserve the section property while eliminating the cancellation of leading terms.

Thus, the only possible nonabelian gauge algebra components that can be realized are

$$
\mathfrak{s u}_{2}, \mathfrak{s u}_{3}, \mathfrak{g}_{2}, \mathfrak{s o}_{7}, \mathfrak{s o}_{8}, \mathfrak{f}_{4}, \mathfrak{e}_{6}, \mathfrak{e}_{7}, \mathfrak{e}_{8}
$$

We can identify explicit examples in which each of these gauge algebras is realized in a non-Higgsable cluster. The simplest set of examples corresponds to the case where the divisor supporting the gauge group is the surface $S=\mathbb{P}^{2}$. In this case, similar to the $6 \mathrm{D}$ case where non-Higgsable gauge groups arise on rational curves $\mathbb{P}^{1}$, all divisors in the surface $S$ are linearly equivalent to a multiple of the hyperplane class $H$, so the relevant line bundles can be classified by a single integer. In particular, the normal bundle can be chosen so that $N=-n H$ for any integer $n$. This local condition can be realized explicitly in the context of a global model by taking a compact base $B_{3}=\tilde{\mathbb{F}}_{n}$ that is a $\mathbb{P}^{1}$ bundle over $\mathbb{P}^{2}$ formed by projectivization of the line bundle $\mathcal{O}(-n H)$. Such geometries were described previously in [58, 64-66]. In this case, the divisors (3.13) and (3.14) become

$$
\begin{aligned}
F_{k} & =(12-(4-k) n) H \\
G_{k} & =(18-(6-k) n) H,
\end{aligned}
$$

where we assume $\phi=\gamma=0$ so that the divisor $S$ does not intersect other divisors on which $f, g$ must vanish, in accord with the assumption that this is an isolated single-factor non-Higgsable cluster. It is straightforward to read off the Kodaira singularity types on $S$ associated with different values of $n$. For example, for $n=4, F_{k}=(4 k-4) H, G_{k}=$ $(4 k-6) H$, so $(f, g)$ vanish to degrees $(1,2)$, corresponding to a type III codimension one singularity supporting an $\mathfrak{s u}_{2}$ gauge algebra. Similarly, for $n=5,6, \ldots, 12$, the associated gauge algebras are $\mathfrak{g}_{2}, \mathfrak{s o}_{8}, \mathfrak{f}_{4}, \mathfrak{f}_{4}, \mathfrak{e}_{6}, \mathfrak{e}_{7}, \mathfrak{e}_{7}, \mathfrak{e}_{7}$, and for $n=18$ the gauge algebra is $\mathfrak{e}_{8}$. For $13 \leq n \leq 17$, there is a codimension two $(4,6)$ locus associated with a curve on $S$, analogous to the $(4,6)$ points on curves of self-intersection $-9,-10,-11$ in the $6 \mathrm{D}$ case. This gives explicit examples of all the single-factor non-Higgsable gauge group possibilities other than $\mathfrak{s u}_{3}$ and $\mathfrak{s o}_{7}$.

While $\mathfrak{s u}_{3}$ cannot be realized as a single-factor non-Higgsable cluster on a divisor $\mathbb{P}^{2}$ that does not intersect other divisors on which $f, g$ vanish, $\mathfrak{s u}_{3}$ can be realized on divisors that realize other types of surfaces. Several explicit examples were given in [5, 58]; in one case, if $S=\mathbb{F}_{0}=\mathbb{P}^{1} \times \mathbb{P}^{1}$, which has $-K=2 S+2 F$ where $S, F$ are the curves associated with the two $\mathbb{P}^{1}$ factors, we can take $N=-3 S-3 F$, and we have

$$
\begin{aligned}
F_{k} & =(3 k-4)(S+F) \\
G_{k} & =(3 k-6)(S+F) .
\end{aligned}
$$


It follows that $f_{0}=f_{1}=g_{0}=g_{1}$, and $g_{2}, \hat{g}_{2} \in \mathcal{O}(0)$, so we have a type IV singularity with no monodromy, and the gauge algebra is $\mathfrak{s u}_{3}$. This geometry can be realized in the context of a $\mathbb{P}^{1}$ bundle over $\mathbb{F}_{0}$, produced from projectivization of a line bundle $\mathcal{O}(3 S+3 F)$.

It may seem naïvely that it should not be possible to realize $\mathfrak{s o}_{7}$ as a single-factor non-Higgsable cluster on any divisor that does not intersect other divisors on which $f, g$ vanish. In this case we must have $f_{2} \in \mathcal{O}(2 X), g_{3} \in \mathcal{O}(3 X)$, where $X=-2 K+2 N$, and one might think that if $2 X$ is effective then so is $3 X$. This is not true, however, for surfaces in threefold bases, illustrating one of the subtle aspects of generalizing the Zariski decomposition to threefolds. The issue is that $-2 K$ may be an effective divisor, while $-3 K$ may only be in the effective cone (e.g. an effective $\mathbb{Q}$-divisor) without a realization as an integer linear combination of irreducible algebraic hypersurfaces.

An explicit example of an isolated $\mathfrak{s o}_{7}$ non-Higgsable cluster that illustrates this phenomenon can be constructed as follows. Consider a set of toric divisors $B, D, F$, $H$ on $\mathbb{F}_{0}$ that each have self-intersection zero and intersect cyclically with intersections $H \cdot B=B \cdot D=D \cdot F=F \cdot H=1$. Now blow the surface up at these four intersection points giving exceptional divisors $A, C, E, G$. We then have a toric surface $S$ with a set of toric divisors $A-H$ having self-intersections $(-1,-2,-1,-2,-1,-2,-1,-2)$. The anti-canonical class is

$$
-K=A+B+C+D+E+F+G+H
$$

and there are two equivalence relations (from the Stanley-Reisner ideal)

$$
A+B+C \sim E+F+G, \quad C+D+E \sim G+H+A .
$$

Now, we consider embedding this surface into a threefold with normal bundle

$$
N=-C-2 D-4 E-3 F-3 G-H .
$$

We can then write

$$
-4 K+2 N=B+D+F+H,
$$

which is clearly effective, so $f_{2}$ is generically nonzero. In this geometry, however, the divisor $-6 K+3 N=3 A+3 B+3 H-3 E$, while in integer homology cannot be written as a sum of effective irreducible divisors with nonnegative integer coefficients. Thus, in this case $\hat{g}_{3}$ vanishes, $\hat{f}_{2}$ is not a perfect square, and we have an isolated $\mathfrak{s o}_{7}$ non-Higgsable cluster. This local geometry can be realized globally by simply considering a $\mathbb{P}^{1}$ bundle over the surface $S$ with an appropriate twist $T=-N$. An explicit computation of the orders of vanishing of $f, g$ on the divisors in this threefold base using toric methods confirms the presence of a non-Higgsable $\mathfrak{s o}_{7}[58]$.

\section{$5.2 \quad$ Matter}

While in $6 \mathrm{D}$ the only single gauge factor that can have associated non-Higgsable matter is $\mathfrak{e}_{7}, 4 \mathrm{D}$ constructions can provide a much richer range of matter associated with isolated non-Higgsable gauge factors. The primary reason for this difference is that while the 
possible classes of zero-cycles that could support a codimension two matter locus for a $6 \mathrm{D}$ theory are labeled by a single integer (the degree), in $4 \mathrm{D}$, the codimension two matter is supported on curves, and there can be many topologically distinct curve classes within a single surface that could each in principle support matter.

In fact, there is no clear a priori bound on the number of curves that may support matter associated with any given kind of gauge group factor on a complex surface of sufficiently complicated topology. In principle, a complex surface could have a very large number of mutually disjoint complex curves, each appearing in the anti-canonical class and each supporting matter. We expect that there is a bound on the set of surface types $S$ that can arise as divisors in complex threefold bases $B_{3}$, analogous to the bound on surfaces that can act as bases for elliptically fibered Calabi-Yau threefolds. We do not, however, have an explicit statement about the existence of such a bound. Note that surfaces $S$ that cannot act as bases $B_{2}=S$ for an elliptically fibered Calabi-Yau threefold can nonetheless arise as divisors in a base $B_{3}$; for example, in the example above of $B_{3}$ a $\mathbb{P}^{1}$ bundle over $\mathbb{P}^{2}$, with $n=18$, one of the divisors associated with a curve in the base $\mathbb{P}^{2}$ has the form of a Hirzebruch surface $\mathbb{F}_{18}$, which cannot support an elliptically fibered Calabi-Yau threefold.

In general, localized matter appears on a curve $C$ within a divisor $D$ in $B_{3}$ when the Kodaira singularity type on $C$ exceeds that of generic points on $D$. For each gauge algebra type, for generic coefficients in the Weierstrass model, this occurs when certain coefficients $f_{k}, g_{k}$ vanish. For example, on a divisor carrying a type $\operatorname{III}^{*}\left(\mathfrak{e}_{7}\right)$ codimension one singularity, matter will be localized along the vanishing locus of $\hat{f}_{3}$, where the $(3,5)$ III* singularity is enhanced to a $(4,5) \mathrm{II}^{*}$ singularity. ${ }^{3}$ Like the $6 \mathrm{D}$ case of the -7 curve that carries an $\mathfrak{e}_{7}$ gauge algebra and matter in the $\frac{1}{2} \mathbf{5 6}$ representation, a divisor $D \cong \mathbb{P}^{2} \subset B_{3}$ with a normal bundle of $N=-10 H$ or $-11 H$ carries an $\mathfrak{e}_{7}$ algebra; the matter locus is determined by $\hat{f}_{3} \in \Gamma(\mathcal{O}(12 H+N))$, so the geometric matter lies on a conic or pair of lines in the case $N=-10 H$, and on a single line in the class $H$ when $N=-11 H$.

As an example of a situation where multiple curves support geometric matter in a nonHiggsable cluster, consider the case where the divisor $D=\mathrm{dP}_{3}$ is a del Pezzo surface formed by blowing up $\mathbb{P}^{2}$ at three points, giving three exceptional curves $E_{i}$ with $E_{i} \cdot E_{i}=-1$, and three lines $L_{i j}=H-E_{i}-E_{j}$. The anti-canonical class is $-K=3 H-E_{1}-E_{2}-E_{3}=$ $L_{12}+L_{13}+L_{23}+E_{1}+E_{2}+E_{3}$. If we take the normal bundle of $D$ to be the line bundle associated with the divisor $N=2 E_{1}-4 L_{12}-4 E_{2}-6 L_{23}$, it is straightforward to verify that the gauge algebra on $D$ is $\mathfrak{e}_{7}$, and

$$
f_{3} \in \Gamma(\mathcal{O}(-4 K+N))=\Gamma\left(\mathcal{O}\left(2 H+2 E_{1}+2 E_{2}+2 E_{3}\right)\right) .
$$

It follows that matter is supported on the three disjoint curves $E_{i}$. This local geometry can be embedded in a global threefold base $B_{3}$ that is a $\mathbb{P}^{1}$ bundle over $\mathrm{dP}_{3}$, by simply taking $B_{3}$ to be the projectivization of the line bundle $N$. This example is one of the complete set of possible $\mathbb{P}^{1}$ bundles over del Pezzo and generalized del Pezzo surfaces that were classified and studied in [58].

\footnotetext{
${ }^{3}$ The "Kodaira type" along the matter curve is simply the type associated with the specified order of vanishing. As observed in $[40,41]$, this does not imply that the resolved Calabi-Yau manifold has a fiber of that particular type in the codimension two locus.
} 
Similar examples of non-Higgsable gauge groups associated with geometric matter on one or more curves can be constructed for other choices of gauge algebra. Two examples of this type with a non-Higgsable $\mathfrak{s u}_{3}$ realized through a type IV singularity are described explicitly in [5]. In one of these examples, for instance, $B_{3}$ is a $\mathbb{P}^{1}$ bundle over $\mathrm{dP}_{2}$ with $N=E_{1}+E_{2}-L_{12}$, which gives a type IV singularity over the section $\Sigma_{-}$, which is itself a $\mathrm{dP}_{2}$ with normal bundle $N$. We have

$$
g_{2} \in \Gamma(\mathcal{O}(-6 K+4 N))=\Gamma\left(\mathcal{O}\left(2 L_{12}\right)\right) .
$$

Since $L_{12}=H-E_{1}-E_{2}$ is a -1 curve, and hence a rigid divisor within the surface $\Sigma_{-}$, this means that $g_{2}$ vanishes to order 2 on $L_{12}$ and is a perfect square. So the gauge algebra is $\mathfrak{s u}_{3}$, and matter is localized on the curve $L_{12}$.

Other examples in which multiple curves within a divisor $D$ carry matter can arise in situations where $D$ itself carries a gauge group but also intersects with multiple other divisors that also carry nonabelian gauge group factors. We describe some explicit examples of this kind in the following sections.

\section{Products of two factors}

We now consider situations where a pair of divisors $D_{a}$ and $D_{b}$ intersect on a curve $C_{a b}$ and both carry non-Higgsable gauge groups. Because the order of vanishing of $f, g$ on $C_{a b}$ is at least the sum of that on $D_{a}, D_{b}$, the same is true for $g$, and the minimal orders of vanishing that give a non-Higgsable gauge group factor are $(1,2)$, we cannot have a product containing anything larger than a type $\mathrm{I}_{0}^{*}(2,3)$ singularity. Furthermore, as noted above and in appendix $\mathrm{A}$, an $\mathfrak{s o}_{7}$ algebra can only arise when the orders of vanishing are $(2,4)$, which would lead to a $(4,6)$ singularity when combined with an $\mathfrak{s u}_{3}$ component. This restricts us to the eight possibilities $\mathfrak{s u}_{2} \oplus \mathfrak{s u}_{2}, \mathfrak{s u}_{2} \oplus \mathfrak{s u}_{3}, \mathfrak{s u}_{3} \oplus \mathfrak{s u}_{3}, \mathfrak{g}_{2} \oplus \mathfrak{s u}_{2}, \mathfrak{g}_{2} \oplus \mathfrak{s u}_{3}$, $\mathfrak{s o}_{7} \oplus \mathfrak{s u}_{2}, \mathfrak{s o}_{8} \oplus \mathfrak{s u}_{2}, \mathfrak{s o}_{8} \oplus \mathfrak{s u}_{3}$.

Some simple examples of product groups can be found by taking bases of the form $B_{3}=\mathbb{P}^{1} \times B_{2}$ where $B_{2}$ contains a $(-2,-3)$ non-Higgsable cluster; in these cases $B_{3}$ has a non-Higgsable cluster associated with the lifts of the corresponding divisors, with gauge algebra $\mathfrak{s u}_{2} \oplus \mathfrak{g}_{2}$; note that the generic monodromy condition is not modified in this case as the available set of Weierstrass monomials simply increases in the product space.

Examples with a gauge algebra $\mathfrak{s u}_{2} \oplus \mathfrak{s u}_{3}$, corresponding to the nonabelian part of the gauge group of the standard model of particle physics, were described in [5].

In [67], a systematic analysis of all models where the base $B_{3}$ is a $\mathbb{P}^{1}$ bundle over one of the toric bases $B_{2}$ from [57] will be described. These models contain a wide range of examples of non-Higgsable gauge groups, including examples of five possible two-factor combinations:

$$
\begin{array}{rlr}
\mathfrak{s u}_{2} \oplus \mathfrak{s u}_{2}, & \mathfrak{s u}_{2} \oplus \mathfrak{s u}_{3}, & \mathfrak{s u}_{3} \oplus \mathfrak{s u}_{3}, \\
\mathfrak{g}_{2} \oplus \mathfrak{s u}_{2}, & \mathfrak{s o}_{7} \oplus \mathfrak{s u}_{2} &
\end{array}
$$

In fact, these are the only possible algebras associated with two-factor gauge products. All other possibilities can be ruled out by a local analysis. 
Considering the remaining possibilities in turn, first assume that there exist divisors $A, B$ that have nonzero intersection and that support gauge algebra factors $\mathfrak{s u}_{3} \oplus \mathfrak{s o}_{8}$ or $\mathfrak{s u}_{3} \oplus \mathfrak{g}_{2}$, with the $\mathfrak{s u}_{3}$ supported on $A$. We can choose local coordinates so that $z=0$ on $A$ and $w=0$ on $B$. Then there must be a leading term in $g$ of the form $z^{2} w^{3}$. If there were no such term than we would have a $(4,6)$ singularity on $A \cap B$. If such a term exists, however, then $g /\left.z^{2}\right|_{z=0}$ cannot be a perfect square. So $A$ cannot support a non-Higgsable $\mathfrak{s u}_{3}$, giving a contradiction. It follows that neither $\mathfrak{s u}_{3} \oplus \mathfrak{s o}_{8}$ nor $\mathfrak{s u}_{3} \oplus \mathfrak{g}_{2}$ can be realized in a non-Higgsable cluster.

Now assume that $A, B$ intersect and support non-Higgsable algebra factors $\mathfrak{s u}_{2} \oplus \mathfrak{s o}_{8}$. Using the same coordinate system as above, the singularity on $A$ cannot be type IV, since there would again be a leading term in $g$ of the form $z^{2} w^{3}$, and $g /\left.w^{3}\right|_{w=0}$ would not be a perfect cube. If there is no term in $g$ of the form $z^{2} w^{3}$ and we have a type III singularity on $A$, then there must be a leading term in $f$ of the form $z w^{2}$. But then $f /\left.w^{2}\right|_{w=0}$ cannot be a perfect square, so $B$ cannot support a non-Higgsable $\mathfrak{s o}_{8}$. Again we have a contradiction, so there is no non-Higgsable gauge group containing a connected pair of factors with algebra $\mathfrak{s u}_{2} \oplus \mathfrak{s o}_{8}$.

Thus, all two-factor products are ruled out in non-Higgsable clusters except for those having the five algebras listed in (6.1).

\section{More complicated "quiver diagrams"}

While in $6 \mathrm{D}$, there are only two possible gauge algebras with multiple components that can arise from a non-Higgsable cluster, in 4D the structure is much richer. As in the case of matter, this occurs because while all points on a curve represent the same element of homology, on a surface there can be many distinct curves that are mutually non-intersecting and are non-homologous. A given divisor that carries a non-Higgsable gauge group factor can thus intersect with many other divisors, each of which carries a non-Higgsable gauge group factor of its own, along a set of distinct curves.

This means that there is no obvious constraint that limits the number of gauge factors that a given non-Higgsable gauge group factor can be connected to through (geometric) matter in 4D models. The set of connected gauge group factors in the non-Higgsable cluster can thus contain "branchings" where a single gauge group factor is connected to three or more other factors. Furthermore, since a gauge factor can in general be connected to two other factors, with no constraint other than the limit on pairings from (6.1), it is possible to have long chains connecting one branching point to another, or to itself, which could in principle produce graphs of arbitrary complexity in the absence of some global bound.

The graphs describing non-Higgsable clusters for 4D theories are conveniently described by the standard diagrammatic convention of "quivers", discussed in the physics literature in [68]. In a quiver diagram, each gauge group factor is represented by a node in a graph, and a directed arrow from a group factor $G$ to a group factor $H$ corresponds to matter in a bifundamental representation $\left(R_{G}, \bar{R}_{H}\right)$. Since we are here only focused on the geometric aspect of the gauge groups and matter involved, all matter will be represented by bidirectional arrows, corresponding to $\mathcal{N}=2$ type matter in the $4 \mathrm{D}$ theory. 
The local rules that we have derived here do not seem to place any significant constraints on the complexity of quivers that can arise from non-Higgsable geometries in F-theory constructions of $4 \mathrm{D}$ vacua. It seems likely that, as for Calabi-Yau threefolds, the number of distinct topological classes of in elliptically fibered Calabi-Yau fourfolds is finite and hence that there are some actual bounds on the complexity of possible quivers from compact threefold bases $B_{3}$. We leave a global analysis of these issues for future work. Here, we simply give a few examples in which the branching and chain features just mentioned are realized explicitly.

\subsection{Branchings}

An explicit example of a base $B_{3}$ that gives a non-Higgsable cluster exhibiting branching can be constructed as follows. We begin with a toric base $B_{2}$ chosen from the bases computed in [57], characterized by a sequence of toric divisors (curves) $C_{1}, \ldots, C_{9}$ with self-intersections $-n_{i}$

$$
\left(-n_{1}, \ldots,-n_{9}\right)=(2,-3,-1,-3,-1,-4,-1,-4,0) .
$$

We then construct $B_{3}$ as a $\mathbb{P}^{1}$ bundle over $B_{2}$ from the projectivization of the line bundle $N=\sum_{i} a_{i} C_{i}$, where

$$
\left(-a_{1}, \ldots,-a_{9}\right)=(0,0,-1,0,1,1,3,2,5) .
$$

While the non-Higgsable cluster associated with this base can be worked out in principle using the methods we have derived here, in practice for a toric base like this it is generally easier to simply analyze the orders of vanishing of $f, g$ on the various divisors using the toric approach, which is easily automated. To do this, the divisors $C_{i}$ and the sections $\Sigma_{ \pm}$are represented as rays $v_{i}$ in $N=\mathbb{Z}^{3}$. The monomials in the Weierstrass model are then the elements of the dual lattice $M=N^{*}$ that satisfy $\left\langle m, v_{i}\right\rangle \geq-4,-6$ for $f, g$ respectively. The orders of vanishing on any divisor or curve can be determined by simply considering the set of available monomials. In this case, carrying out this analysis shows that there is a type III singularity on $\Sigma_{-}$carrying a $\mathfrak{s u}_{2}$ gauge algebra, and type IV, $\mathrm{I}_{0}^{*}$, and $\mathrm{I}_{0}^{*}$ singularities on $C_{4}, C_{6}, C_{8}$ carrying gauge factors $\mathfrak{s u}_{2}, \mathfrak{g}_{2}$, and $\mathfrak{g}_{2}$. The branched quiver diagram representing this non-Higgsable cluster is depicted in figure 1 . Note that for this particular construction, there are no codimension three points where $f, g$ vanish to orders 4, 6. Many constructions exhibiting branching do have such codimension three points. Further examples of non-Higgsable clusters exhibiting branching appear in the full set of $\mathbb{P}^{1}$ bundles over $B_{2}$ 's from [57], and will be described further in [67].

\subsection{Chains}

In six dimensions, there is only one situation (the $\mathfrak{s u}_{2} \oplus \mathfrak{s o}_{7} \oplus \mathfrak{s u}_{2}$ cluster) in which a nonHiggsable cluster contains a gauge factor that intersects (carries jointly charged matter with) more than one other gauge factor. In four dimensions, however, this can happen in a variety of ways, as discussed above. In particular, there are many local configurations in which a non-Higgsable gauge group can be supported on a divisor $S$ that intersects two 


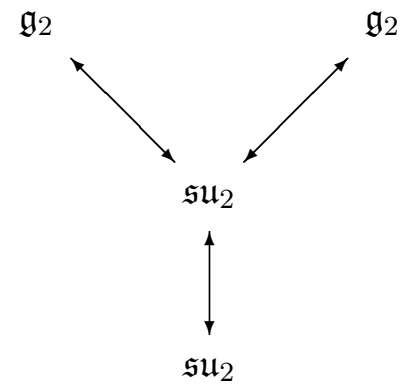

Figure 1. The quiver diagram associated with a non-Higgsable cluster having gauge algebra $\mathfrak{s u}_{2} \oplus \mathfrak{s u}_{2} \oplus \mathfrak{g}_{2} \oplus \mathfrak{g}_{2}$, with bifundamental (geometrically non-chiral) matter connecting the first $\mathfrak{s u}_{2}$ component with the other three gauge factors.

other divisors $S^{\prime}, S^{\prime \prime}$ that both carry non-Higgsable gauge groups themselves. This opens the possibility of a long linear chain of connected gauge factors in a non-Higgsable cluster. Such chains cannot be ruled out by the local analysis we have presented here. Furthermore, some exploration of the space of possible configurations shows that there are global models in which relatively long non-Higgsable chains of this kind can arise.

As an explicit example, we demonstrate how it is possible in a simple class of toric models to realize non-Higgsable clusters with gauge algebras of the form $\mathfrak{s u}_{2} \oplus \mathfrak{s u}_{3} \oplus \mathfrak{s u}_{3} \oplus$ $\cdots \mathfrak{s u}_{3} \oplus \mathfrak{s u}_{2}$, where the number of $\mathfrak{s u}_{3}$ summands can go up to at least 11 .

Conceptually, the idea of the examples we describe here is that we can take a set of surfaces $S_{i}$ in $B_{3}$ to be a chain of $\mathrm{dP}_{3}$ del Pezzo surfaces, each with normal bundle $N=-K$. The surfaces will be connected so that, for example, $C_{i}=S_{i} \cap S_{i+1}$ will be the curve $E_{1}$ in $S_{i}$ and $L_{23}$ in $S_{i+1}$, using the notation of section 5.2. On each surface in such a sequence we have $f_{0}, g_{0} \in \Gamma(\mathcal{O}(0))$. If we blow up a curve on one such surface, it forces $f_{0}, g_{0}$ to vanish on that surface. This contributes to $\phi, \gamma$ on the adjoining surfaces, where $f_{0}, g_{0}$ must also vanish, etc.

To realize this explicitly in a $3 \mathrm{D}$ toric base $B_{3}$, consider the following construction: we start with $\mathbb{P}^{1} \times \mathbb{F}_{m}, m \leq 8$, considered as a trivial $\mathbb{P}^{1}$ bundle over $\mathbb{F}_{m}$, and denote by $\tilde{S}, F$ the divisors given by the lift of curves in the base that are in the classes of the section with self-intersection $+m$ and the fiber. We blow up on the curve $\tilde{S} \cap F$, giving an exceptional divisor $E_{1}$; we repeat, blowing up on the curve $E_{1} \cap \tilde{S}$, then on the new curve $E_{2} \cap \tilde{S}$ with $E_{2}$ the new exceptional divisor, etc., for a total of $2 m$ times. We then blow up the curves $E_{2} \cap \Sigma_{-}, \ldots, E_{2 m-2} \cap \Sigma_{-}$in ascending order, and the curves $E_{2 m-2} \cap \Sigma_{+}, \ldots, E_{2} \cap \Sigma_{+}$in descending order, where $\Sigma_{ \pm}$are two sections of the original trivial $\mathbb{P}^{1}$ bundle, giving further exceptional divisors $E_{i}^{\prime}, E_{i}^{\prime \prime}$ for $i \in\{2, \ldots, 2 m-2\}$. This geometry contains within it a linear chain of $2 m-3$ del Pezzo surfaces $\mathrm{dP}_{3}$ with normal bundles $N=-K$ as described above; these are the proper transforms of the surfaces $E_{2}, \ldots, E_{2 m-2}$. We then blow up the curve $E_{2} \cap E_{2}^{\prime}$. This leads to $(4,6)$ singularities over $E_{i} \cap E_{i}^{\prime}, E_{i} \cap E_{i-1}^{\prime}$ for $i=3, \ldots, 2 m-4$, which are resolved by blowing up these curves. The final geometry has no $(4,6)$ divisors or curves when $m \leq 8$, and can be analyzed most easily by toric methods to have a nonHiggsable gauge algebra $\mathfrak{s u}_{2} \oplus \mathfrak{s u}_{3} \oplus \cdots \oplus \mathfrak{s u}_{3} \oplus \mathfrak{s u}_{2}$ on the chain of divisors $E_{2} \ldots, E_{2 m-2}$. In 
the case $m=8$, we thus have a chain of 13 non-Higgsable gauge group factors, including 11 copies of $\mathfrak{s u}_{3}$. For $8<m<12$, the situation becomes more complicated as an $\mathfrak{e}_{8}$ singularity with $(4,6)$ matter curves develops on the surfaces $S, \tilde{S}$; we do not analyze the details of these geometries here.

This construction is most easily visualized in the toric language, where the toric divisors can be defined through the fan

$$
\begin{aligned}
& \left(\Sigma_{ \pm}\right) \quad v_{1,2}=(0,0, \pm 1) \\
& (\tilde{S}) \quad v_{3}=(0,1,0) \\
& v_{4}=(1,0,0) \\
& \text { (S) } \quad v_{5}=(0,-1,0) \\
& \text { (F) } \quad v_{6}=(-1,-m, 0) \\
& \left(E_{i}\right) \quad v_{6+i}=(-1,-m+i, 0), \quad 1 \leq i \leq 2 m \\
& \left(E_{i}^{\prime}\right) \quad v_{5+2 m+i}=(-1,-m+i, 1), \quad 2 \leq i \leq 2 m-2 \\
& \left(E_{i}^{\prime \prime}\right) \quad v_{2+6 m-i}=(-1,-m+i,-1), \quad 2 \leq i \leq 2 m-2 \\
& v_{6 m-1+i}=(-2,-2 m+2 i, 1), \quad 2 \leq i \leq 2 m-4 \\
& v_{8 m-6+i}=(-2,-2 m+2 i+1,1), \quad 2 \leq i \leq 2 m-5
\end{aligned}
$$

The triangulation associated with the cone structure for this fan is shown schematically in figure 2 for the case $m=4$.

This family of examples illustrates the possibility that long chains of connected gauge group factors can arise in a 4D non-Higgsable cluster geometry.

\subsection{Loops}

Another complication that can arise in a 4D non-Higgsable cluster is the presence of a closed loop in the quiver diagram. Locally, such a loop looks much like the chains described in the previous subsection, and there is no way to rule out such loops based on purely local considerations.

We have identified a number of examples where a loop arises in a non-Higgsable cluster geometry. In one simple example, there is a loop consisting of four $\mathfrak{s u}_{2}$ factors, so that the total gauge algebra is $\mathfrak{s u}_{2} \oplus \mathfrak{s u}_{2} \oplus \mathfrak{s u}_{2} \oplus \mathfrak{s u}_{2}$, with matter curves supporting matter in the bifundamental of each adjacent pair of gauge group factors, as well as between the initial and final factors. This example can be constructed as follows: beginning with $\mathbb{P}^{1} \times \mathbb{P}^{1} \times \mathbb{P}^{1}$, with divisors $X_{ \pm}, Y_{ \pm}, Z_{ \pm}$associated with two distinct points on each of the three $\mathbb{P}^{1}$ 's, and labeling $D_{i}=Y_{+}, X_{+}, Y_{-}, X_{-}$for $i=1,2,3,4$, we first blow up the curves $Z_{ \pm} \cap D_{i}$, giving new exceptional divisors $E_{i \pm}$. We then blow up on the curves $E_{i+} \cap D_{i}$. Blowing up four more $(4,6)$ curves gives us a good F-theory base with no $(4,6)$ divisors, curves, or points. In the final geometry, the proper transforms of the initial divisors $D_{i}$ each carry an $\mathfrak{s u}_{2}$ gauge factor, and they are connected in a cyclic chain as described above. This can all be done in the toric language, starting with the fan spanned by the rays $v_{1}=(0,0,+1)$, $v_{2}=(0,0,-1), v_{3}=(0,1,0), v_{4}=(1,0,0), v_{5}=(0,-1,0), v_{6}=(-1,0,0)$, corresponding to $Z_{+}, Z_{-}, Y_{+}, X_{+}, Y_{-}, X_{-}$, and then blowing up on the appropriate edges of the toric 
$\mathfrak{s u}_{2} \longleftrightarrow \mathfrak{s u}_{3} \longleftrightarrow \mathfrak{s u}_{3} \longleftrightarrow \mathfrak{s u}_{3} \longleftrightarrow \mathfrak{s u}_{2}$

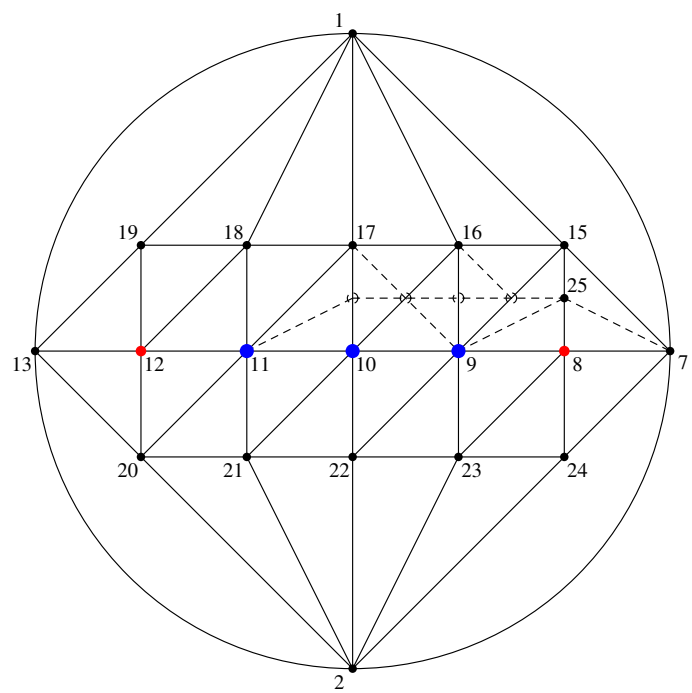

(B)

Figure 2. A global model with a non-Higgsable geometry giving rise to a linear chain in the quiver structure of the gauge algebra, $\mathfrak{s u}_{2} \oplus \mathfrak{s u}_{3} \oplus \mathfrak{s u}_{3} \oplus \mathfrak{s u}_{3} \oplus \mathfrak{s u}_{2}$. (A) The quiver diagram of the chain, (B) a schematic depiction of the triangulation of the $3 \mathrm{D}$ toric fan describing the global geometry, formed from a sequence of blowups at points $7\left(E_{1}\right), \ldots$, from an initial fan describing the threefold $\mathbb{P}^{1} \times \mathbb{F}_{4}$. (Note that points associated with the toric rays $v_{3}-v_{6}$ and $v_{14}$ are not shown.) Large blue dots represent divisors supporting an $\mathfrak{s u}_{3}$ gauge summand, smaller red dots are divisors supporting an $\mathfrak{s u}_{2}$ gauge algebra. Open circles represent $(4,6)$ curves that must be blown up to divisors after the blow-ups up to $v_{25}$. Note that before blowing up to $v_{25}$, the divisors associated with points $9,10,11$ are connected del Pezzo $\mathrm{dP}_{3}$ surfaces (as can be seen from the structure of solid lines). Similar constructions are possible with up to (at least) 11 factors of $\mathfrak{s u}_{3}$ in the linear chain.

fan in the sequence described above. A schematic picture of the triangulation of the final fan, with vertices labeled in the order of blowups, is given in figure 3.

\section{Conclusions}

\subsection{Summary and open questions}

We have initiated a systematic analysis of geometric non-Higgsable clusters that can arise in threefolds $B_{3}$ for F-theory compactifications that give $\mathcal{N}=1$ supergravity theories in four dimensions. These structures describe gauge groups and matter that cannot be Higgsed at the geometric level by deformation of complex structure moduli, and which therefore arise at generic points in the moduli space of the corresponding Calabi-Yau fourfold. More work must be done to ascertain whether these geometrically non-Higgsable structures are truly present in the low-energy supergravity theory of any specific F-theory vacuum. The presence of G-flux, the corresponding superpotential, and extra degrees of freedom not yet incorporated systematically into F-theory may affect this conclusion. Unless one of these factors causes a generic change in qualitative behavior, however, it seems that the non-Higgsable structures we have analyzed here will be generic features of F-theory vacua. 


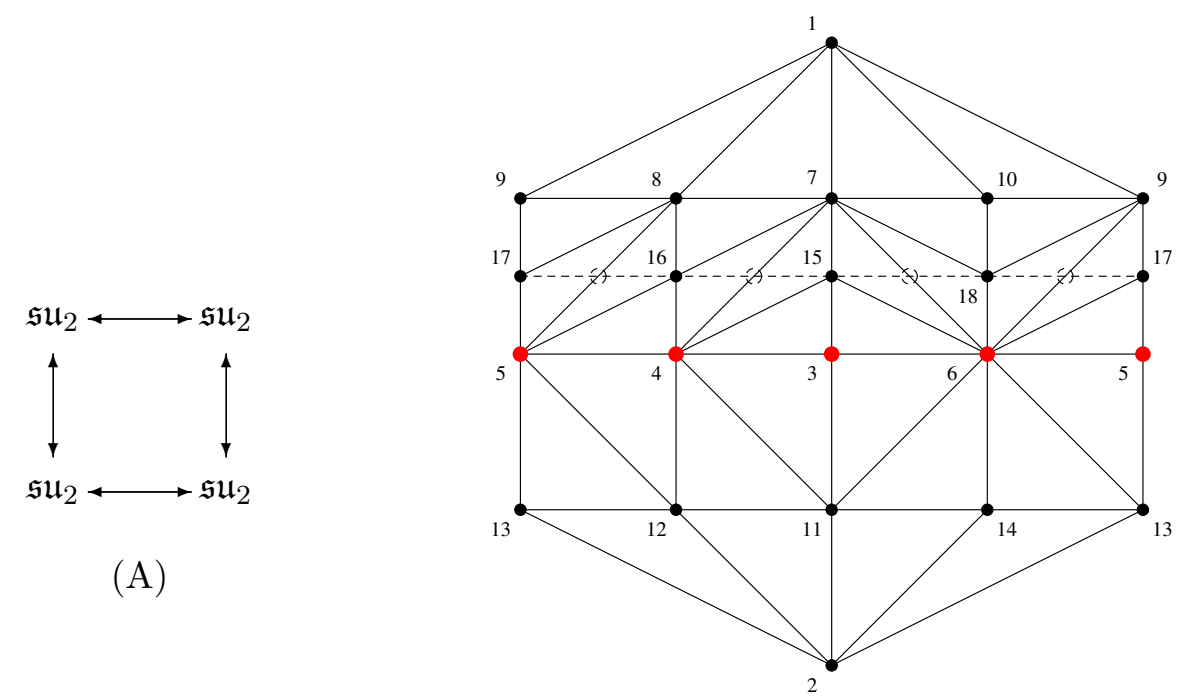

(B)

Figure 3. A global model with a non-Higgsable geometry giving rise to a loop in the quiver structure of the gauge algebra. (A) The quiver diagram of the loop, (B) a schematic depiction of the triangulation of the 3D toric fan describing the global geometry, formed from a sequence of blowups at points $7, \ldots$, from an initial fan describing the threefold $\mathbb{P}^{1} \times \mathbb{P}^{1} \times \mathbb{P}^{1}$. (Note that the points on the left should be identified with the points on the right with the same labels.)

We have developed a set of local equations that govern the possible non-Higgsable structures that can appear on a combination of divisors in the F-theory base manifold. For toric bases, a straightforward monomial analysis can be used to determine non-Higgsable structures in any specific case.

The set of individual gauge factors that can arise in a non-Higgsable cluster is quite limited, and contains only nine distinct possible simple Lie algebras. Similarly, the number of products of two gauge factors that can arise is also quite limited, and consists of only five possibilities, including that of the nonabelian part of the standard model $\mathrm{SU}(3) \times$ $\mathrm{SU}(2)$, which was analyzed specifically in [5]. Unlike in 6D, however, the topological structure of the gauge groups in a non-Higgsable cluster, which can be depicted in a quiver diagram, can apparently be quite complicated. From the local analysis there is no constraint that prohibits branching, loops, or long linear chains of gauge factors. Indeed, we have identified explicit global geometries that contain each of these three features. An interesting open question is the extent to which global constraints limit the complexity of the graph structures that can arise for large non-Higgsable clusters. Indeed, since unlike for elliptically fibered Calabi-Yau threefolds, there is as yet no proof that the number of distinct topological types of elliptically fibered Calabi-Yau fourfolds is finite, there is no clear argument at this time that would bound the size of possible non-Higgsable clusters for $4 \mathrm{D}$ F-theory compactifications.

One issue that we have not addressed here is whether geometries with codimension three $(4,6)$ singularities at points need to be treated in any special way. Such singularities cannot be blown up without tuning additional complex structure moduli. These therefore do not represent additional branches in the space of fourfolds, unlike $(4,6)$ singularities in 
codimension two. Like codimension two $(4,6)$ singularities, however, which correspond to superconformal field theories coupled to gravity, the codimension three singularities may also represent some kind of more exotic gravitationally coupled theory.

\subsection{Classifying Calabi-Yau fourfolds}

The classification of non-Higgsable clusters on 2D bases [4] has enabled a systematic analysis of the space of elliptic Calabi-Yau threefolds, giving a fairly clear global picture of the space of $6 \mathrm{D}$ F-theory models $[57,69-72]$. It is hoped that the results presented here will similarly provide a useful tool for exploring the space of 3D bases for F-theory compactifications to four dimensions. Such analysis of elliptic Calabi-Yau fourfolds, however, will be significantly more complicated than the corresponding analysis for elliptic Calabi-Yau threefolds. For elliptic Calabi-Yau threefolds, the minimal model program [73] and the work of Grassi [62] give a simple characterization of the set of possible bases as blowups of the Hirzebruch surfaces $\mathbb{F}_{m}, m \leq 12, \mathbb{P}^{2}$, and the Enriques surface. For elliptic Calabi-Yau fourfolds, where the base is a complex threefold, no such simple characterization of minimal bases is known; constructing such a classification using the minimal model approach (Mori theory) in higher dimensions is an interesting open problem. The analysis of non-Higgsable clusters given here may provide some guidance in attempting to systematically address this problem.

One physical motivation for attempting a systematic global characterization of the space of possible elliptic Calabi-Yau fourfolds and associated non-Higgsable clusters is the goal of identifying generic features or specific constraints that F-theory places on $4 \mathrm{D}$ supergravity theories. In six dimensions, F-theory geometry places various constraints on the possible effective theories that can be realized. Some of these constraints are understood in the physical theory as anomaly constraints, while other constraints place additional consistency conditions on the low-energy theory [6, 74]. F-theory geometry also places strong constraints on the compactification geometry that have manifestations in the lowenergy 4D supergravity theory. Some initial exploration of such constraints was carried out in $[58,66]$, but it seems likely that as our understanding of the global space of F-theory vacua matures further insights into the constraints produced on low-energy theories will emerge.

The approach of analyzing elliptic fibrations through the geometry of the base provides a complementary approach to the long-studied toric approach to describing Calabi-Yau manifolds as hypersurfaces in toric varieties pioneered by Batyrev [75] and the complete intersection (CICY) approach taken in [76]. While both these approaches give a large class of Calabi-Yau manifolds, and can be used to study elliptic Calabi-Yau's (see e.g. [77-83], the classification of bases using non-Higgsable clusters is in principle both a simpler approach as it reduces the complexity of the geometry involved, and a more complete approach as it is in principle possible to describe all elliptic Calabi-Yau's from this point of view. We expect that the complementary insights provided by these different approaches, all of which are currently under active investigation, will provide many new insights into the geometry of elliptic Calabi-Yau fourfolds in the near future. 


\subsection{Physical consequences of non-Higgsable clusters}

At the present time, F-theory represents one of the most general approaches to constructing vacuum solutions of string theory for which analytic tools are available. While, unlike in $6 \mathrm{D}, \mathrm{F}$-theory in its current formulation does not seem to in any sense cover the full space of $4 \mathrm{D}$ string vacua, the vacua formed from F-theory seem to represent a much larger and broader sample than those $4 \mathrm{D}$ vacua constructed from many other approaches. For example, F-theory vacua with a smooth heterotic dual are a small subset of the full set of possible F-theory constructions [58]. And since the Hodge numbers of generic elliptic Calabi-Yau fourfolds are much larger than those of threefolds, the number of flux vacua formed from F-theory constructions is estimated to be much much larger than those from other constructions such as IIB flux vacua [7]. On the other hand, the apparently infinite number of topologically distinct type IIA flux vacua [84] and the potentially even greater number of non-geometric string compactifications may provide even larger families of vacua than those realized through standard F-theory compactifications. F-theory also, however, provides a window on the nonperturbative dynamics of string vacua in a way that is not available from other string constructions that require a weak coupling limit. Given these observations, it seems that F-theory gives one of the best pictures we have so far for the behavior of a "generic" class of nonperturbative string vacua. Assuming that issues such as G-flux do not substantially change the structure of the gauge factors that are forced by non-Higgsable clusters in the F-theory geometry, a suggestive picture emerges of the physics of a "typical" F-theory vacuum in the landscape. In particular, as emphasized also in [5], non-Higgsable clusters provide a mechanism that may make light matter fields and gauge symmetries a natural consequence of generic string compactifications, without requiring any special tuning.

Non-Higgsable structures seem to be highly prevalent in F-theory vacua. In six dimensions, of the more than 100,000 possible base manifolds studied in [57,70] that support elliptic Calabi-Yau threefolds, only 27 lack non-Higgsable gauge groups. Typical elliptic threefolds with large Hodge numbers have many non-Higgsable clusters, with numerous factors of the gauge algebras $\mathfrak{e}_{8}, \mathfrak{f}_{4}, \mathfrak{g}_{2} \oplus \mathfrak{s u}_{2}$. We expect a similar story to hold for fourfolds, though the types of factors that are typical in $4 \mathrm{D}$ models has not yet been systematically analyzed. In particular, we expect that for fourfolds with large Hodge numbers, which are likely to give rise to the largest number of distinct flux vacua, there will typically be many non-Higgsable gauge group factors. Some initial systematic investigation in this direction will be presented in [67].

While the possible structures that can arise in non-Higgsable clusters for 4D models may be quite complicated, the set of possible gauge groups, and in particular the products of two gauge groups that can appear in these clusters, is actually quite limited. Specifically, the nonabelian part of the standard model gauge group, $\mathrm{SU}(3) \times \mathrm{SU}(2)$, is one of only five possible two-summand Lie algebra structures that can arise from non-Higgsable clusters. If we assume that matter, and at least two nonabelian gauge factors, are the minimal necessary components for "interesting" (i.e., anthropic) physics in the landscape, then the nonabelian part of the standard model arises as simply one of five natural minimal possibil- 
ities that may arise throughout the landscape. While this is certainly suggestive, and may provide an alternative framework for F-theory phenomenology to the well-studied F-theory GUT approach [85-89], many significant questions must be answered to provide realistic models of particle physics from this approach. The abelian $U(1)$ factor in the standard model, for example, must either be tuned by hand, or must also arise in a non-Higgsable fashion. While the latter possibility has been shown to be possible in 6D models [70, 90], such a mechanism is not yet well understood in four dimensions. It is also necessary to understand better how geometric non-Higgsability relates to the field theory description of the low-energy theory, and for a more realistic model further structure such as the Yukawa couplings would need to be computed in any specific geometry with the proper gauge groups and matter content. Note that while of course the $\mathrm{SU}(2)$ of the standard model seen in nature is broken by the Higgs field, this could in principle occur even in a $4 \mathrm{D}$ F-theory model with the $\mathrm{SU}(2)$ in a non-Higgsable cluster, if there is an appropriate geometrically non-Higgsable matter field charged under the $\mathrm{SU}(2)$ that acquires a negative mass through radiative corrections after SUSY is broken; further discussion and analysis of the non-Higgsable $\mathfrak{s u}_{3} \oplus \mathfrak{s u}_{2}$ structure appears in [5].

If we assume that all nonabelian gauge groups and matter arising in nature come from generic (i.e. non-Higgsable) structures, then the structure of non-Higgsable gauge group factors would also place interesting constraints on dark matter. One possibility for dark matter is a non-Higgsable cluster (or multiple clusters) with one or more gauge group factors that are completely disconnected from the standard model; such disconnected dark matter sectors have also been considered in the F-theory GUT literature [87]. In the context of non-Higgsable clusters, this would correspond to hidden sector dark matter with specific possible gauge groups and matter content. In the simplest cases, this could be simply an additional supersymmetric $\mathfrak{s u}_{2}, \mathfrak{s u}_{3}, \mathfrak{g}_{2}, \mathfrak{s o}_{7}, \mathfrak{s o}_{8}, \mathfrak{f}_{4}, \mathfrak{e}_{6}, \mathfrak{e}_{7}$ or $\mathfrak{e}_{8}$ sector with a spectrum of glueballs that would interact only gravitationally with ordinary matter. Another possibility for dark matter can arise if the nonabelian standard model components $\mathrm{SU}(3) \times \mathrm{SU}(2)$ lie in a non-Higgsable cluster, but with other gauge factors connected in the quiver diagram. For example, the standard model factors could arise as part of a non-Higgsable cluster with gauge algebra $\mathrm{SU}(3) \times \mathrm{SU}(2) \times G$, with additional matter charged under the $\mathrm{SU}(2)$ and $G$ factors; this would correspond to a weakly interacting dark matter sector. An interesting consequence is that if such a dark matter sector arises from a non-Higgsable cluster then it would have to be associated with an internal gauge group $G$ that would be restricted to have one of the gauge algebras $\mathfrak{s u}_{2}, \mathfrak{s u}_{3}, \mathfrak{g}_{2}$, or $\mathfrak{s o}_{7}$. In fact, these are the only possibilities for gauge algebras that can connect to the $\mathfrak{s u}_{2}$ of the standard model in any non-Higgsable cluster. The discovery of matter charged under a hidden gauge group in this family would thus fit naturally with the predictions of a generic F-theory model. On the other hand, discovery of weakly interacting matter with, for example, an additional $\mathrm{SU}(N)$ gauge group sector where $N>3$ would rule out the hypothesis that the low-energy spectrum seen in nature arises from non-Higgsable geometric structures in generic F-theory models.

Combining these features, the simplest picture of the "typical" F-theory vacuum that we have available at this time would be a model where the visible light fields consist of one or more single or multi-factor gauge groups with or without matter, with only five 
possibilities including $\mathrm{SU}(3) \times \mathrm{SU}(2)$ for two-factor visible gauge group products carrying jointly charged matter. There could also be dark matter sectors corresponding to other additional, completely decoupled, non-Higgsable clusters, or additional sectors that come from the same cluster and that could be charged for example under an $\mathrm{SU}(2)$ in an $\mathrm{SU}(3) \times$ $\mathrm{SU}(2)$ product as well as another hidden gauge group that would have to have the algebra $\mathfrak{s u}_{2}, \mathfrak{s u}_{3}, \mathfrak{g}_{2}$, or $\mathfrak{s o}_{7}$ as just discussed above. Clearly, supersymmetry breaking, which we have completely ignored here, would need to be incorporated in any realistic model. This would also give rise to possible pseudoscalar axion-like fields from the lifting of scalar moduli from the supersymmetric model. The structure of light fields that we see in the observed universe is not too different from this highly simplified picture of what we might expect from a generic F-theory vacuum. At our current state of understanding, this picture of the typical F-theory vacuum is still quite incomplete and cannot yet be used to make specific predictions for low-energy non-supersymmetric physics. While the analysis of geometric non-Higgsable clusters in this paper is based on rigorous mathematical reasoning, the connection between the underlying geometry and low-energy physics is not as direct in 4D F-theory models as in six dimensions, where the low-energy physics precisely mirrors the geometry. Much more work must be done to understand the role of G-flux and 7-brane world-volume degrees of freedom in F-theory, and to incorporate supersymmetry breaking into supersymmetric 4D F-theory vacuum models. It seems possible, however, that even as our understanding improves the non-Higgsable clusters described here may continue to play an important and perhaps predictive role in describing the generic properties of $4 \mathrm{D}$ supersymmetric vacua of F-theory.

\section{Acknowledgments}

We would like to thank Lara Anderson, Mboyo Esole, Antonella Grassi, Jim Halverson, Jonathan Heckman, Sam Johnson, Shamit Kachru, Shu-Heng Shao, Eva Silverstein, Tracy Slatyer, and Yinan Wang for helpful discussions and the referee for helpful comments. We would also like to thank the Aspen Center for Physics, where the initial stages of this work were carried out. The research of W.T. is supported by the U.S. Department of Energy under grant Contract Number DE-SC00012567. The research of D.R.M. is supported by by the National Science Foundation under grant PHY-1307513. We thank the Aspen Center for Physics for hospitality and partial support by the National Science Foundation Grant No. PHYS-1066293. 


\section{A The gauge algebra of maximally Higgsed models}

In this appendix we discuss the monodromy conditions for F-theory seven-branes of type $\mathrm{I}_{0}^{*}$. If $D=\{z=0\}$ describes the location of the brane (in local coordinates), then $f, g$, and $\Delta$ have orders $\geq 2, \geq 3$, and 6 along $D$, respectively. We let $\hat{f}=\left.\left(f / z^{2}\right)\right|_{\{z=0\}}$, $\hat{g}=\left.\left(g / z^{3}\right)\right|_{\{z=0\}}$ and $\hat{\Delta}=\left.\left(\Delta / z^{6}\right)\right|_{\{z=0\}}$ (all in the local coordinate chart). These are sections of line bundles

$$
\mathcal{O}_{D}\left(-2 k K_{B}-k N_{D / B}\right)=\mathcal{O}_{D}\left(-2 k K_{D}+k N_{D / B}\right)
$$

for $k=2,3,6$, respectively.

The monodromy and gauge algebra are now determined by the behavior of the cubic polyomial

$$
x^{3}+\hat{f} x+\hat{g} .
$$

- If there are sections $\alpha, \beta \in \Gamma\left(\mathcal{O}_{D}\left(-2 K_{D}+N_{D / B}\right)\right)$ such that $\hat{f}=-\left(\alpha^{2}+\alpha \beta+\beta^{2}\right)$ and $\hat{g}=\alpha \beta(\alpha+\beta)$, then

$$
x^{3}+\hat{f} x+\hat{g}=(x-\alpha)(x-\beta)(x+\alpha+\beta)
$$

and the gauge algebra is $\mathfrak{s o}(8)$. To ensure that the seven-brane has type $\mathrm{I}_{0}^{*}$, we also need

$$
\hat{\Delta}=-(\alpha-\beta)^{2}(2 \alpha+\beta)^{2}(\alpha+2 \beta)^{2}
$$

to be not identically zero. That is, three things must be avoided:

(i) $\beta=\alpha$, which would imply $\hat{f}=-3 \alpha^{2}$ and $\hat{g}=2 \alpha^{3}$.

(ii) $\beta=-2 \alpha$, which would imply $\hat{f}=-3 \alpha^{2}$ and $\hat{g}=2 \alpha^{3}$.

(iii) $\beta=-\frac{1}{2} \alpha$, which would imply $\hat{f}=-\frac{3}{4} \alpha^{2}, \hat{g}=-\frac{1}{4} \alpha^{3}$.

- If there are sections $\lambda \in \Gamma\left(\mathcal{O}_{D}\left(-2 K_{D}+N_{D / B}\right)\right)$ and $\mu \in \Gamma\left(\mathcal{O}_{D}\left(-4 K_{D}+2 N_{D / B}\right)\right)$ such that $\lambda^{2}-4 \mu$ is not the square of a section of $\mathcal{O}_{D}\left(-2 K_{D}+N_{D / B}\right)$ and such that $\hat{f}=\mu-\lambda^{2}$ and $\hat{g}=-\lambda \mu$, then

$$
x^{3}+\hat{f} x+\hat{g}=(x-\lambda)\left(x^{2}+\lambda x+\mu\right)
$$

and the gauge algebra in $\mathfrak{s o}(7)$. To ensure that the seven-brane has type $\mathrm{I}_{0}^{*}$, we also need

$$
\hat{\Delta}=\left(\mu+2 \lambda^{2}\right)^{2}\left(4 \mu-\lambda^{2}\right)
$$

to not vanish identically.

- In all other cases, the gauge algebra is $\mathfrak{g}_{2}$.

We point out two particular ways to solve these constraints (although these solutions are not the most general ones possible). 
Solution 1. If the spaces of sections $\Gamma\left(\mathcal{O}_{D}\left(-2 K_{D}+N_{D / B}\right)\right), \Gamma\left(\mathcal{O}_{D}\left(-4 K_{D}+2 N_{D / B}\right)\right)$ and $\Gamma\left(\mathcal{O}_{D}\left(-6 K_{D}+3 N_{D / B}\right)\right)$, are all one-dimensional and $\hat{u} \in \Gamma\left(\mathcal{O}_{D}\left(-2 K_{D}+N_{D / B}\right)\right)$ is not identically zero, then there are constants $A$ and $B$ so that $\hat{f}=A \hat{u}^{2}$ and $\hat{g}=B \hat{u}^{3}$. There are then constants $r_{1}, r_{2}, r_{3}$ such that the constant polynomial $X^{3}+A X+B$ can be factored into linear factors:

$$
X^{3}+A X+b=\prod_{i=1}^{3}\left(X-r_{i}\right)
$$

and there is a corresponding factorization of a section of $\mathcal{O}_{D}\left(-6 K_{D}+3 N_{D / B}\right)$, namely,

$$
x^{3}+\hat{f} x+\hat{g}=\prod_{i=1}^{3}\left(x-r_{i} u\right) .
$$

In this case, we get gauge algebra $\mathfrak{s o}(8)$ for any choice of $\hat{f}$ and $\hat{g}$ provided that $\hat{\Delta} \not \equiv 0$.

Solution 2. If $\hat{g} \equiv 0$ and $\hat{f}$ is not a square, then the gauge algebra must be $\mathfrak{s o}(7)$. For in this case, we can set $\lambda=0, \mu=\hat{f}$ to satisfy the criterion given above. Note that $\hat{\Delta}=4 \mu^{3} \not \equiv 0$ since $\mu$ is not a square.

We now consider what monodromies can occur in the case of an F-theory seven-brane of type $I_{0}^{*}$ in a model which has been maximally Higgsed. Fixing the base $B$ and the divisor $D$ at which the seven-brane is located, there are restriction maps

$$
\rho_{k}: \quad \Gamma\left(\mathcal{O}_{B}\left(-2 k K_{B}+k D\right)\right) \rightarrow \Gamma\left(\mathcal{O}_{D}\left(-2 k K_{D}+k N_{D}\right)\right)
$$

(identifying $\mathcal{O}_{D}(D)$ with $\mathcal{O}_{D}\left(N_{D}\right)$ ). If $f$ and $g$ are generic, i.e., the model is maximally Higgsed, then $\hat{f}$ will be a generic element of the image of $\rho_{2}$, while $\hat{g}$ will be a generic element of the image of $\rho_{3}$. Here is how we will use the "maximally Higgsed" property: if we scale $(\hat{f}, \hat{g}) \rightarrow\left(c_{1} \hat{f}, c_{2} \hat{g}\right)$, we should obtain the same gauge algebra for general constants $c_{1}, c_{2}$. (If not, then further Higgsing is possible by scaling $f$ and $g$.)

Consider first the case that the gauge algebra is $\mathfrak{s o}(8)$, and let $\alpha, \beta \in \Gamma\left(\mathcal{O}_{D}\left(-2 K_{D}+\right.\right.$ $\left.\left.N_{D / B}\right)\right)$ be sections such that $\hat{f}=-\left(\alpha^{2}+\alpha \beta+\beta^{2}\right)$ and $\hat{g}=\alpha \beta(\alpha-\beta)$. Suppose that $\alpha$ and $\beta$ are linearly independent in the complex vector space $\Gamma\left(\mathcal{O}_{D}\left(-2 K_{D}+N_{D / B}\right)\right)$. We first remark that this implies that $\left\{\alpha^{2}, \alpha \beta, \beta^{2}\right\}$ are linearly independent in $\Gamma\left(\mathcal{O}_{D}\left(-4 K_{D}+\right.\right.$ $\left.\left.2 N_{D / B}\right)\right)$ and that $\left\{\alpha^{2} \beta, \alpha \beta^{2}\right\}$ are linearly independent in $\Gamma\left(\mathcal{O}_{D}\left(-6 K_{D}+3 N_{D / B}\right)\right)$. This is because a linear dependence relation among powers of degree $N, \sum K_{j} \alpha^{j} \beta^{N-j} \equiv 0$ with constant coefficients $K_{j}$, would lead to a linear dependence relation among powers of degree 1 by factoring the homogeneous polynomial $\sum K_{j} \xi^{j} \eta^{N-j} \in \mathbb{C}[\xi, \eta]$ into homogeneous linear factors and choosing a factor which vanishes upon subtituting $\alpha$ for $\xi$ and $\beta$ for $\eta$.

Since $\alpha$ and $\beta$ are assumed to be linearly independent, none of $\alpha, \beta$, and $\alpha+\beta$ can vanish identically. Thus, the locus $\{\hat{g}=0\}$ must decompose as a union of $\{\alpha=0\},\{\beta=0\}$, and $\{\alpha+\beta=0\}$. After generic scaling $(\hat{f}, \hat{g}) \rightarrow\left(c_{1} \hat{f}, c_{2} \hat{g}\right)$ we get the same locus:

$$
\left\{c_{2} \hat{g}=0\right\}=\{\hat{g}=0\}
$$


Thus, in order to get the same kind of decomposition, after permuting $\{\alpha, \beta,-\alpha-\beta\}$ if necessary (which can be achieved with a linear transformation on the span of $\alpha$ and $\beta$ ), we can assume that the sections $\alpha^{\prime}$ and $\beta^{\prime}$ that are needed after scaling take the form

$$
\alpha^{\prime}=c_{3} \alpha ; \quad \beta^{\prime}=c_{4} \beta .
$$

Now we get several equations from the linear independence of $\left\{\alpha^{2}, \alpha \beta, \beta^{2}\right\}$ and $\left\{\alpha^{2} \beta, \alpha \beta^{2}\right\}$. Since $c_{1}\left(\alpha^{2}+\alpha \beta+\beta^{2}\right)=c_{3}^{2} \alpha^{2}+c_{3} c_{4} \alpha \beta+c_{4}^{2} \beta^{2}$, we see that $c_{1}=c_{3}^{2}=c_{3} c_{4}=c_{4}^{2}$. On the other hand, since $c_{2}\left(\alpha^{2} \beta+\alpha \beta^{2}\right)=c_{3}^{2} c_{4} \alpha^{2} \beta+c_{3} c_{4}^{2} \alpha \beta^{2}$, we see that $c_{2}=c_{3}^{2} c_{4}=c_{3} c_{4}^{2}$. It follows that $c_{2}^{2}=c_{3}^{3} c_{4}^{3}=c_{1}^{3}$ which does not hold for general $c_{1}, c_{2}$.

The conclusion is that $\alpha$ and $\beta$ must in fact be linearly dependent in the complex vector space $\Gamma\left(\mathcal{O}_{D}\left(-2 K_{D}+N_{D / B}\right)\right)$, so both of them can be written as constant multiples of a section $\hat{u}$. It follows that $\hat{f}=A \hat{u}^{2}$ and $\hat{g}=B \hat{u}^{3}$ for some constants $A$ and $B$.

Suppose that $\operatorname{dim} \Gamma\left(\mathcal{O}_{D}\left(-2 K_{D}+N_{D / B}\right)\right)>1$. Then not every element of $\Gamma\left(\mathcal{O}_{D}\left(-4 K_{D}+2 N_{D / B}\right)\right)$ is the square of an element of $\Gamma\left(\mathcal{O}_{D}\left(-2 K_{D}+N_{D / B}\right)\right)$, so the generic $\hat{f}$ is not of the form $A \hat{u}^{2}$ for any $\hat{u}$. Thus. if the gauge algebra is $\mathfrak{s o}(8)$, $\operatorname{dim} \Gamma\left(\mathcal{O}_{D}\left(-2 K_{D}+N_{D / B}\right)\right)$ must be 1.

Suppose that $\operatorname{dim} \Gamma\left(\mathcal{O}_{D}\left(-2 K_{D}+N_{D / B}\right)\right)=1$ with generator $\hat{u}$, but $\operatorname{dim} \Gamma\left(\mathcal{O}_{D}\left(-4 K_{D}+2 N_{D / B}\right)\right)>1\left(\right.$ respectively $\left.\operatorname{dim} \Gamma\left(\mathcal{O}_{D}\left(-6 K_{D}+3 N_{D / B}\right)\right)>1\right)$. Then the generic element of $\Gamma\left(\mathcal{O}_{D}\left(-4 K_{D}+2 N_{D / B}\right)\right)$ does not have the form $A \hat{u}^{2}$ (respectively, the generic element of $\Gamma\left(\mathcal{O}_{D}\left(-6 K_{D}+3 N_{D / B}\right)\right)$ does not have the form $\left.B \hat{u}^{3}\right)$, so the maximally Higgsed gauge algebra is not $\mathfrak{s o}(8)$.

It follows that any maximally Higgsed model with $\mathfrak{s o}(8)$ gauge symmetry must take the form of Solution 1 above.

Consider now the case that the gauge algebra is $\mathfrak{s o}(7)$, and let $\lambda \in \Gamma\left(\mathcal{O}_{D}\left(-2 K_{D}+\right.\right.$ $\left.\left.N_{D / B}\right)\right), \mu \in \Gamma\left(\mathcal{O}_{D}\left(-4 K_{D}+2 N_{D / X}\right)\right)$ be sections such that $\hat{f}=\mu-\lambda^{2}$ and $\hat{g}=-\lambda \mu$. We cannot have $\mu \equiv 0$ or else $\hat{f}$ would be a perfect square and the gauge algebra would be $\mathfrak{s o}(8)$. So $\mu \not \equiv 0$. If in addition $\lambda \not \equiv 0$, then the locus $\{\hat{g}=0\}$ is the union of $\{\lambda=0\}$ and $\{\mu=0\}$. Thus, if we scale the coefficients $(\hat{f}, \hat{g}) \rightarrow\left(c_{1} \hat{f}, c_{2} \hat{g}\right)$, the new sections $\lambda^{\prime}$ and $\mu^{\prime}$ must satisfy $\lambda^{\prime}=c_{3} \lambda, \mu^{\prime}=c_{4} \mu$. It follows that $c_{2}=c_{3} c_{4}$ and $\left(c_{1}-c_{4}\right) \mu-\left(c_{1}-c_{3}^{2}\right) \lambda^{2}=0$. If $\mu$ and $\lambda^{2}$ are linearly independent, then $c_{1}=c_{4}=c_{3}^{2}$ which implies that $c_{2}^{2}=c_{3}^{2} c_{4}^{2}=c_{1}^{3}$, but this is not true for general $c_{1}, c_{2}$. Thus, $\mu$ and $\lambda^{2}$ must be linearly dependent and so $\lambda^{2}=K \mu$ (since $\left.\mu \not \equiv 0\right)$. But if $K \neq 0$, then $\mu-\lambda^{2}=\left(\frac{1}{K}-1\right) \lambda^{2}$ is a perfect square, which would force the gauge algebra to be $\mathfrak{s o}(8)$. Thus, $K$ must be 0 and $\lambda$ must vanish identically, and we have solution 2 as above. Thus, we find that a non-Higgsable $\mathfrak{s o}_{7}$ is only possible when $\hat{g}_{3}=0$ identically, and $\hat{f}_{2}$ is not a perfect square. This is only possible for generic choices of $\hat{f}_{2}$ when either $\hat{f}_{2}$ contains only a single non-even monomial in a local coordinate system, or contains multiple independent monomials. Note that this implies that for a maximally Higgsed $\mathfrak{s o}_{7}$ algebra, $g$ must vanish to order at least 4 (rather than order 3) along the gauge divisor.

Open Access. This article is distributed under the terms of the Creative Commons Attribution License (CC-BY 4.0), which permits any use, distribution and reproduction in any medium, provided the original author(s) and source are credited. 


\section{References}

[1] C. Vafa, Evidence for F-theory, Nucl. Phys. B 469 (1996) 403 [hep-th/9602022] [InSPIRE].

[2] D.R. Morrison and C. Vafa, Compactifications of F-theory on Calabi-Yau threefolds. I, Nucl. Phys. B 473 (1996) 74 [hep-th/9602114] [INSPIRE].

[3] D.R. Morrison and C. Vafa, Compactifications of F-theory on Calabi-Yau threefolds. II, Nucl. Phys. B 476 (1996) 437 [hep-th/9603161] [INSPIRE].

[4] D.R. Morrison and W. Taylor, Classifying bases for 6D F-theory models, Central Eur. J. Phys. 10 (2012) 1072 [arXiv:1201.1943] [inSPIRE].

[5] A. Grassi, J. Halverson, J. Shaneson and W. Taylor, Non-Higgsable QCD and the standard model spectrum in F-theory, JHEP 01 (2015) 086 [arXiv:1409.8295] [INSPIRE].

[6] V. Kumar, D.R. Morrison and W. Taylor, Global aspects of the space of $6 D \mathcal{N}=1$ supergravities, JHEP 11 (2010) 118 [arXiv:1008.1062] [INSPIRE].

[7] F. Denef, Les Houches lectures on constructing string vacua, arXiv:0803.1194 [INSPIRE].

[8] W. Taylor, Lectures on D-branes, gauge theory and M(atrices), in High energy physics and cosmology, Proceedings of 1997 Summer School, ICTP, Trieste Italy (1997) [hep-th/9801182] [INSPIRE].

[9] W. Taylor and M. Van Raamsdonk, Multiple D0-branes in weakly curved backgrounds, Nucl. Phys. B 558 (1999) 63 [hep-th/9904095] [INSPIRE].

[10] W. Taylor and M. Van Raamsdonk, Multiple Dp-branes in weak background fields, Nucl. Phys. B 573 (2000) 703 [hep-th/9910052] [INSPIRE].

[11] R.C. Myers, Dielectric branes, JHEP 12 (1999) 022 [hep-th/9910053] [INSPIRE].

[12] R. Donagi, S. Katz and E. Sharpe, Spectra of D-branes with Higgs vevs, Adv. Theor. Math. Phys. 8 (2005) 813 [hep-th/0309270] [INSPIRE].

[13] S. Cecotti, C. Cordova, J.J. Heckman and C. Vafa, T-branes and monodromy, JHEP 07 (2011) 030 [arXiv: 1010.5780] [inSPIRE].

[14] R. Donagi and M. Wijnholt, Gluing branes - I, JHEP 05 (2013) 068 [arXiv:1104.2610] [INSPIRE].

[15] L.B. Anderson, J.J. Heckman and S. Katz, T-branes and geometry, JHEP 05 (2014) 080 [arXiv:1310.1931] [INSPIRE].

[16] M.R. Douglas, D-branes and matrix theory in curved space, Nucl. Phys. Proc. Suppl. 68 (1998) 381 [hep-th/9707228] [InSPIRE].

[17] M.R. Douglas, A. Kato and H. Ooguri, D-brane actions on Kähler manifolds, Adv. Theor. Math. Phys. 1 (1998) 237 [hep-th/9708012] [INSPIRE].

[18] F. Ferrari, On matrix geometry and effective actions, Nucl. Phys. B 871 (2013) 181 [arXiv: 1301.3722] [INSPIRE].

[19] A. Collinucci and R. Savelli, T-branes as branes within branes, arXiv:1410.4178 [INSPIRE].

[20] A. Collinucci and R. Savelli, F-theory on singular spaces, arXiv:1410.4867 [INSPIRE].

[21] J. Marsano, N. Saulina and S. Schäfer-Nameki, Monodromies, fluxes and compact three-generation F-theory GUTs, JHEP 08 (2009) 046 [arXiv:0906.4672] [INSPIRE]. 
[22] R. Blumenhagen, T.W. Grimm, B. Jurke and T. Weigand, Global F-theory GUTs, Nucl. Phys. B 829 (2010) 325 [arXiv:0908.1784] [InSPIRE].

[23] T.W. Grimm, S. Krause and T. Weigand, F-theory GUT vacua on compact Calabi-Yau fourfolds, JHEP 07 (2010) 037 [arXiv:0912.3524] [INSPIRE].

[24] M. Cvetič, T.W. Grimm and D. Klevers, Anomaly cancellation and Abelian gauge symmetries in F-theory, JHEP 02 (2013) 101 [arXiv:1210.6034] [INSPIRE].

[25] J. Marsano and S. Schäfer-Nameki, Yukawas, G-flux and spectral covers from resolved Calabi-Yau's, JHEP 11 (2011) 098 [arXiv:1108.1794] [INSPIRE].

[26] T.W. Grimm and H. Hayashi, F-theory fluxes, chirality and Chern-Simons theories, JHEP 03 (2012) 027 [arXiv:1111.1232] [INSPIRE].

[27] N.C. Bizet, A. Klemm and D.V. Lopes, Landscaping with fluxes and the E 8 Yukawa point in F-theory, arXiv:1404.7645 [INSPIRE].

[28] D.R. Morrison, TASI lectures on compactification and duality, hep-th/0411120 [INSPIRE].

[29] W. Taylor, TASI lectures on supergravity and string vacua in various dimensions, arXiv:1104.2051 [INSPIRE].

[30] N. Nakayama, On Weierstrass models, in Algebraic geometry and commutative algebra, volume II, Kinokuniya (1988), pp. 405-431.

[31] K. Kodaira, On compact analytic surfaces. II, Ann. Math. 77 (1963) 563.

[32] K. Kodaira, On compact analytic surfaces. III, Ann. Math. 78 (1963) 1.

[33] E. Witten, Phase transitions in M-theory and F-theory, Nucl. Phys. B 471 (1996) 195 [hep-th/9603150] [INSPIRE].

[34] P.S. Aspinwall, S.H. Katz and D.R. Morrison, Lie groups, Calabi-Yau threefolds and F-theory, Adv. Theor. Math. Phys. 4 (2000) 95 [hep-th/0002012] [INSPIRE].

[35] M. Bershadsky et al., Geometric singularities and enhanced gauge symmetries, Nucl. Phys. B 481 (1996) 215 [hep-th/9605200] [INSPIRE].

[36] S. Katz, D.R. Morrison, S. Schäfer-Nameki and J. Sully, Tate's algorithm and F-theory, JHEP 08 (2011) 094 [arXiv: 1106.3854] [INSPIRE].

[37] A. Grassi and D.R. Morrison, Anomalies and the Euler characteristic of elliptic Calabi-Yau threefolds, Commun. Num. Theor. Phys. 6 (2012) 51 [arXiv:1109.0042] [inSPIRE].

[38] S.H. Katz and C. Vafa, Matter from geometry, Nucl. Phys. B 497 (1997) 146 [hep-th/9606086] [INSPIRE].

[39] A. Grassi and D.R. Morrison, Group representations and the Euler characteristic of elliptically fibered Calabi-Yau threefolds, J. Algebraic Geom. 12 (2003) 321 [math.AG/0005196] [INSPIRE].

[40] D.R. Morrison and W. Taylor, Matter and singularities, JHEP 01 (2012) 022 [arXiv: 1106.3563] [INSPIRE].

[41] M. Esole and S.-T. Yau, Small resolutions of SU(5)-models in F-theory, Adv. Theor. Math. Phys. 17 (2013) 1195 [arXiv:1107.0733] [INSPIRE].

[42] M. Esole, J. Fullwood and S.-T. Yau, $D_{5}$ elliptic fibrations: non-Kodaira fibers and new orientifold limits of F-theory, arXiv:1110.6177 [INSPIRE]. 
[43] C. Lawrie and S. Schäfer-Nameki, The Tate form on steroids: resolution and higher codimension fibers, JHEP 04 (2013) 061 [arXiv: 1212.2949] [INSPIRE].

[44] A. Grassi, J. Halverson and J.L. Shaneson, Matter from geometry without resolution, JHEP 10 (2013) 205 [arXiv: 1306.1832] [INSPIRE].

[45] H. Hayashi, C. Lawrie, D.R. Morrison and S. Schäfer-Nameki, Box graphs and singular fibers, JHEP 05 (2014) 048 [arXiv: 1402.2653] [INSPIRE].

[46] A. Grassi, J. Halverson and J.L. Shaneson, Non-Abelian gauge symmetry and the Higgs mechanism in F-theory, Commun. Math. Phys. 336 (2015) 1231 [arXiv:1402.5962] [INSPIRE].

[47] M. Esole, S.-H. Shao and S.-T. Yau, Singularities and gauge theory phases, arXiv:1402.6331 [INSPIRE].

[48] N. Seiberg, Nontrivial fixed points of the renormalization group in six-dimensions, Phys. Lett. B 390 (1997) 169 [hep-th/9609161] [INSPIRE].

[49] J.J. Heckman, D.R. Morrison and C. Vafa, On the classification of 6D SCFTs and generalized ADE orbifolds, JHEP 05 (2014) 028 [arXiv: 1312.5746] [INSPIRE].

[50] M. Del Zotto, J.J. Heckman, A. Tomasiello and C. Vafa, 6d conformal matter, JHEP 02 (2015) 054 [arXiv: 1407.6359] [INSPIRE].

[51] K. Ohmori, H. Shimizu, Y. Tachikawa and K. Yonekura, Anomaly polynomial of general $6 d$ SCFTs, Prog. Theor. Exp. Phys. 2014 (2014) 103B07 [arXiv:1408.5572] [InSPIRE].

[52] K. Intriligator, $6 d, \mathcal{N}=(1,0)$ Coulomb branch anomaly matching, JHEP 10 (2014) 162 [arXiv: 1408.6745] [INSPIRE].

[53] B. Haghighat, A. Klemm, G. Lockhart and C. Vafa, Strings of minimal 6d SCFTs, arXiv: 1412.3152 [INSPIRE].

[54] P. Candelas, D.-E. Diaconescu, B. Florea, D.R. Morrison and G. Rajesh, Codimension three bundle singularities in F-theory, JHEP 06 (2002) 014 [hep-th/0009228] [INSPIRE].

[55] S.D. Cutkosky, Zariski decomposition of divisors on algebraic varieties, Duke Math. J. 53 (1986) 149.

[56] W. Fulton, Introduction to toric varieties, Annals of Mathematics Study 131, Princeton University Press, Princeton U.S.A. (1993).

[57] D.R. Morrison and W. Taylor, Toric bases for 6D F-theory models, Fortschr. Phys. 60 (2012) 1187 [arXiv:1204.0283] [INSPIRE].

[58] L.B. Anderson and W. Taylor, Geometric constraints in dual F-theory and heterotic string compactifications, JHEP 08 (2014) 025 [arXiv: 1405.2074] [INSPIRE].

[59] S.H. Katz, D.R. Morrison and M.R. Plesser, Enhanced gauge symmetry in type-II string theory, Nucl. Phys. B 477 (1996) 105 [hep-th/9601108] [INSPIRE].

[60] O.J. Ganor and A. Hanany, Small $E_{8}$ instantons and tensionless noncritical strings, Nucl. Phys. B 474 (1996) 122 [hep-th/9602120] [InSPIRE].

[61] N. Seiberg and E. Witten, Comments on string dynamics in six-dimensions, Nucl. Phys. B 471 (1996) 121 [hep-th/9603003] [INSPIRE].

[62] A. Grassi, On minimal models of elliptic threefolds, Math. Ann. 290 (1991) 287. 
[63] D.R. Morrison and W. Taylor, Sections, multisections and U(1) fields in F-theory, arXiv: 1404.1527 [INSPIRE].

[64] A. Klemm, B. Lian, S.S. Roan and S.-T. Yau, Calabi-Yau fourfolds for M-theory and F-theory compactifications, Nucl. Phys. B 518 (1998) 515 [hep-th/9701023] [INSPIRE].

[65] P. Berglund and P. Mayr, Stability of vector bundles from F-theory, JHEP 12 (1999) 009 [hep-th/9904114] [INSPIRE].

[66] T.W. Grimm and W. Taylor, Structure in $6 D$ and $4 D \mathcal{N}=1$ supergravity theories from F-theory, JHEP 10 (2012) 105 [arXiv: 1204.3092] [INSPIRE].

[67] J. Halverson and W. Taylor, to appear.

[68] M.R. Douglas and G.W. Moore, D-branes, quivers and ALE instantons, hep-th/9603167 [INSPIRE].

[69] W. Taylor, On the Hodge structure of elliptically fibered Calabi-Yau threefolds, JHEP 08 (2012) 032 [arXiv: 1205.0952] [INSPIRE].

[70] G. Martini and W. Taylor, 6D F-theory models and elliptically fibered Calabi-Yau threefolds over semi-toric base surfaces, arXiv:1404.6300 [INSPIRE].

[71] S.B. Johnson and W. Taylor, Calabi-Yau threefolds with large $h^{2,1}$, JHEP 10 (2014) 023 [arXiv: 1406.0514] [INSPIRE].

[72] Y. Wang and W. Taylor, Non-toric bases for 6D F-theory models and elliptic Calabi-Yau threefolds, to appear.

[73] W.P. Barth, K. Hulek, C.A.M. Peters and A. Van de Ven, Compact complex surfaces, Springer (2004).

[74] N. Seiberg and W. Taylor, Charge lattices and consistency of 6D supergravity, JHEP 06 (2011) 001 [arXiv: 1103.0019] [INSPIRE].

[75] V.V. Batyrev, Variations of the mixed Hodge structure of affine hypersurfaces in algebraic tori, Duke Math. J. 69 (1993) 349.

[76] P. Candelas, A.M. Dale, C.A. Lütken and R. Schimmrigk, Complete intersection Calabi-Yau manifolds, Nucl. Phys. B 298 (1988) 493 [inSPIRE].

[77] P. Candelas and A. Font, Duality between the webs of heterotic and type-II vacua, Nucl. Phys. B 511 (1998) 295 [hep-th/9603170] [INSPIRE].

[78] P. Candelas, A. Constantin and H. Skarke, An abundance of K3 fibrations from polyhedra with interchangeable parts, Commun. Math. Phys. 324 (2013) 937 [arXiv:1207.4792] [INSPIRE].

[79] V. Braun, T.W. Grimm and J. Keitel, Geometric engineering in toric F-theory and GUTs with U(1) gauge factors, JHEP 12 (2013) 069 [arXiv: 1306.0577] [INSPIRE].

[80] D. Klevers, D.K. Mayorga Pena, P.-K. Oehlmann, H. Piragua and J. Reuter, F-theory on all toric hypersurface fibrations and its Higgs branches, JHEP 01 (2015) 142 [arXiv:1408.4808] [INSPIRE].

[81] V. Braun, T.W. Grimm and J. Keitel, Complete intersection fibers in F-theory, JHEP 03 (2015) 125 [arXiv: 1411.2615] [INSPIRE].

[82] J. Gray, A.S. Haupt and A. Lukas, All complete intersection Calabi-Yau four-folds, JHEP 07 (2013) 070 [arXiv: 1303.1832] [INSPIRE]. 
[83] J. Gray, A.S. Haupt and A. Lukas, Topological invariants and fibration structure of complete intersection Calabi-Yau four-folds, JHEP 09 (2014) 093 [arXiv: 1405.2073] [INSPIRE].

[84] O. DeWolfe, A. Giryavets, S. Kachru and W. Taylor, Type IIA moduli stabilization, JHEP 07 (2005) 066 [hep-th/0505160] [INSPIRE].

[85] R. Donagi and M. Wijnholt, Model building with F-theory, Adv. Theor. Math. Phys. 15 (2011) 1237 [arXiv:0802.2969] [InSPIRE].

[86] C. Beasley, J.J. Heckman and C. Vafa, GUTs and exceptional branes in F-theory - I, JHEP 01 (2009) 058 [arXiv: 0802.3391] [INSPIRE].

[87] C. Beasley, J.J. Heckman and C. Vafa, GUTs and exceptional branes in F-theory - II. Experimental predictions, JHEP 01 (2009) 059 [arXiv:0806.0102] [INSPIRE].

[88] J.J. Heckman, Particle physics implications of F-theory, Ann. Rev. Nucl. Part. Sci. 60 (2010) 237 [arXiv:1001.0577] [InSPIRE].

[89] T. Weigand, Lectures on F-theory compactifications and model building, Class. Quant. Grav. 27 (2010) 214004 [arXiv: 1009.3497] [INSPIRE].

[90] D.R. Morrison, D. Park and W. Taylor, to appear. 